\title{
Fully automatic cervical vertebrae segmentation framework for X-ray images
}

S. M. Masudur Rahman Al Arif*, a

s.al-arif@city.ac.uk

Karen Knapp

Greg Slabaugh

a Department of Computer Science, City (Please keep this comma, as my University has this in its official name. See the bottom of this page for your reference https://www.city.ac.uk/)University of London, London, UK

buniversity of Exeter Medical School, Exeter, UK

${ }^{*}$ Corresponding author.Fel:: +447960461707

\section{Abstract}

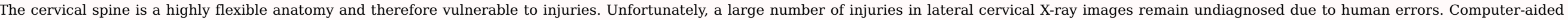

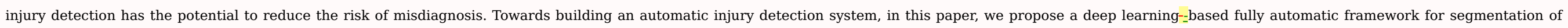

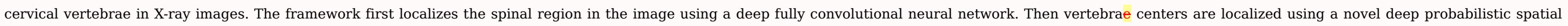

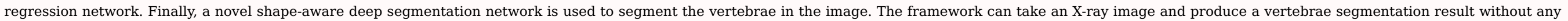

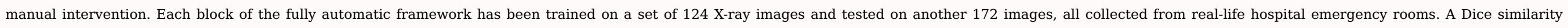
coefficient of 0.84 and a shape error of $1.69 \mathrm{~mm}$ have been achieved.

Keywords: Segmentation; Đeep LearningDeep learning; FCN; UNet; Localization; Cervical vertebrae; X-ray

\section{Introduction}

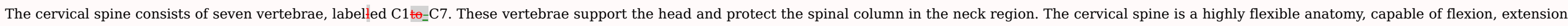

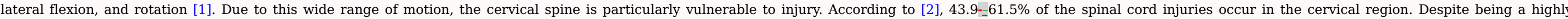

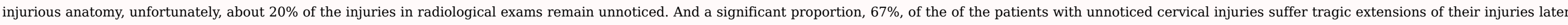
in life [3,4]. Recent developments in the fields of computer vision and artificial intelligence have the potential to reduce the number of missing injuries.

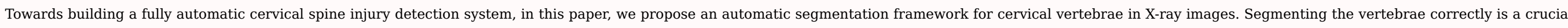

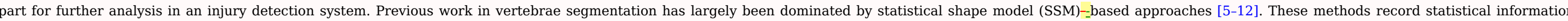

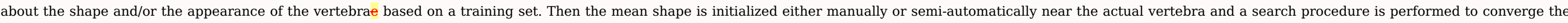
shape on the actual vertebral boundary. Recent literature utilizes random forest--based machine learning models in order to achieve the shape convergence [9-12]

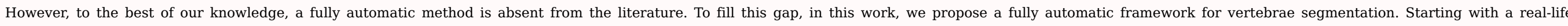

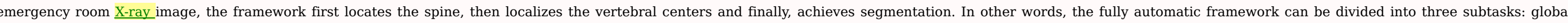
localization, center localization and vertebrae segmentation. Different specialized fully convolutional neural networks (FCN) are used to solve each of these tasks. The complete framework is shown in Fig. 1. 


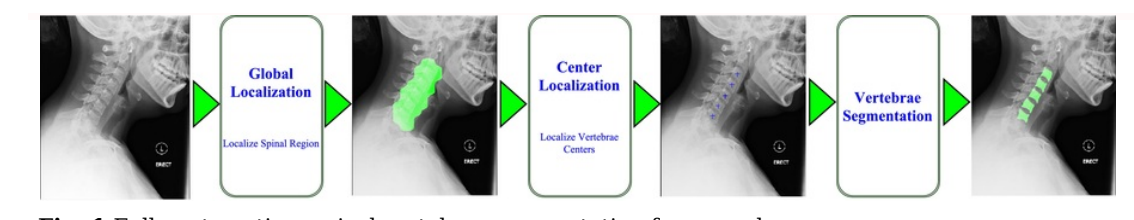

Fig. 1 Fully automatic cervical vertebrae segmentation framework.

alt-text: Fig. 1

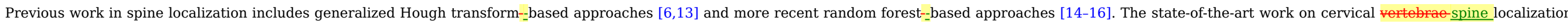

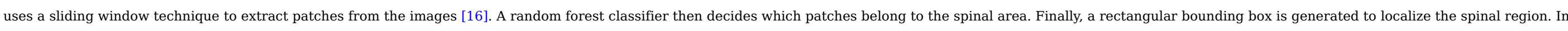

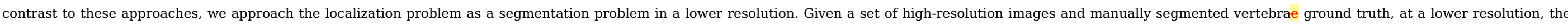

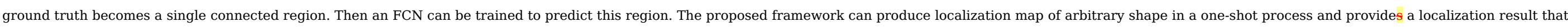
models the cervical spine much better than a rectangular box like [16].

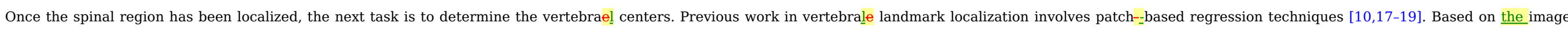

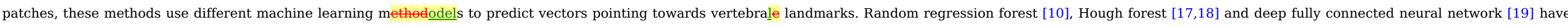

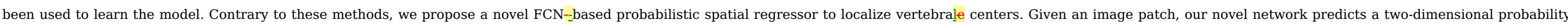
distribution for the localized centers over the patch space. A novel loss function has been introduced to adapt the FCN as a spatial probability predictor.

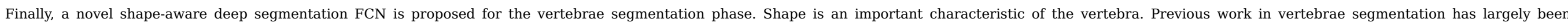

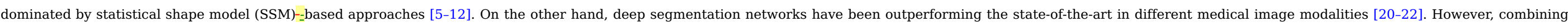
shape information in a deep segmentation network is not straightforward. In this paper, we provide a solution to this problem by introducing a novel shape-aware term in a segmentation loss function.

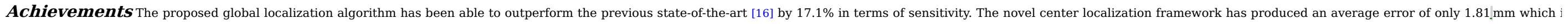

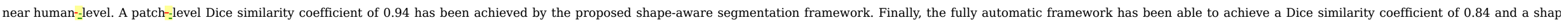
error of $1.69 \mathrm{~mm}$. All these metrics are computed over a challenging dataset of 172 emergency room X-ray images.

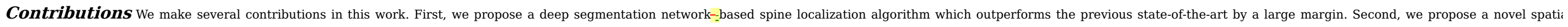

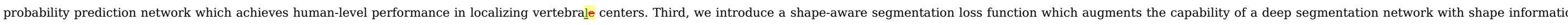

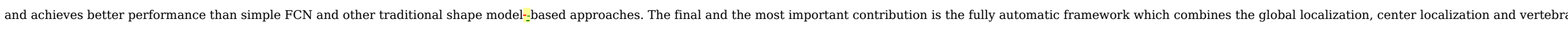
segmentation in a single thread and provides a segmentation result for a real-life emergency room X-ray images without any manual input.

\section{Data}

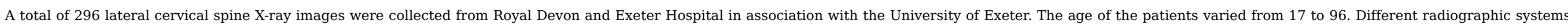

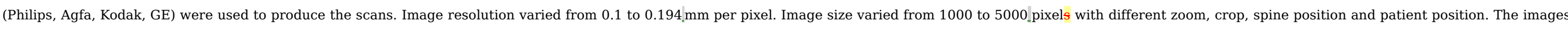

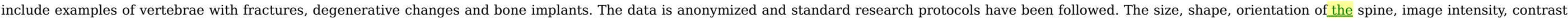

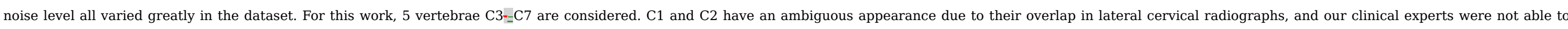

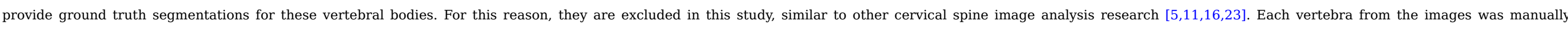
annotated for the vertebral body boundaries and centers by expert radiographers. A few examples with the corresponding manual annotations are shown in Fig. 2 . 


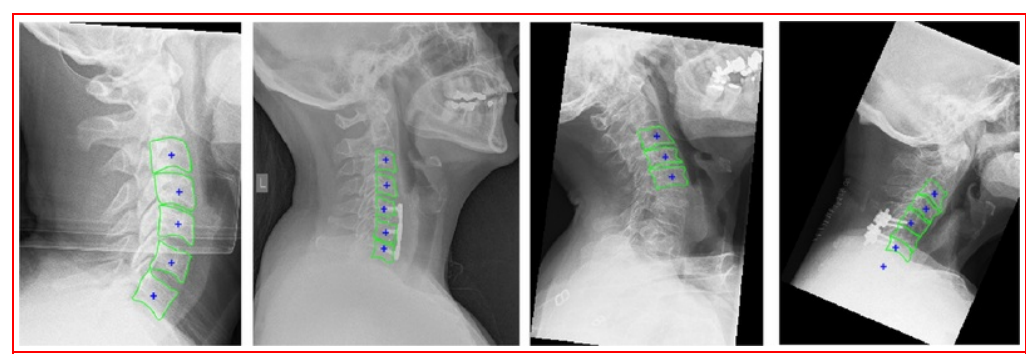

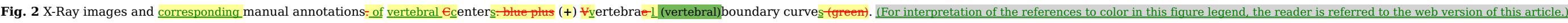
alt-text: Fig. 2

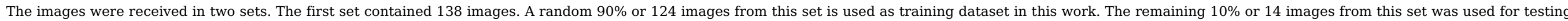
the algorithms. The second set of 158 images were received later into the study and added to the test dataset bringing the total number of test images to 172 .

\section{Global LoealizationGlobal localization}

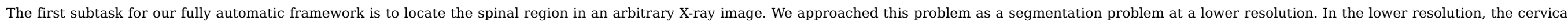
vertebrae become a single connected spinal region. A deep fully convolutional network (FCN) is trained to predict this region.

\subsection{Data}

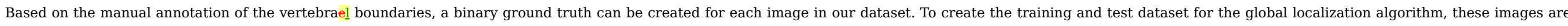

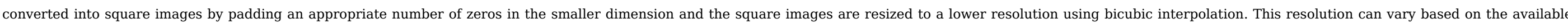

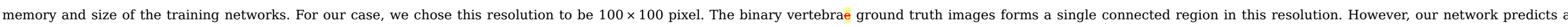

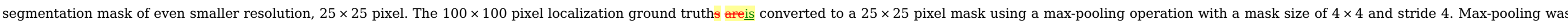
used over interpolation--based methods to keep the localization mask sharp. Fig. 3 shows some of the localization ground truth overlayed on the image after transforming back to the original resolution.

\subsection{Network}

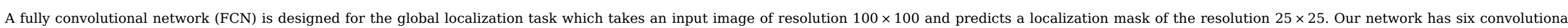

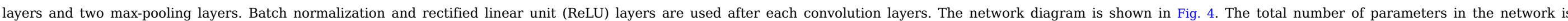
$1,152,450$

\subsection{Training}

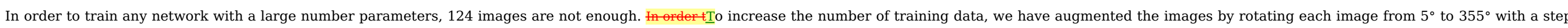

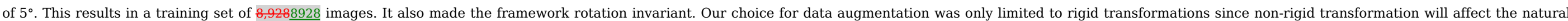
appearance of the spine in the image.

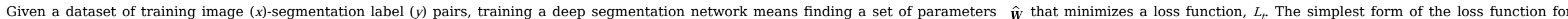
segmentation problem is the pixel-wise log loss or the cross-entropy loss.

$$
\widehat{W}=\underset{\boldsymbol{W}}{\arg \min } \sum_{n=1}^{N} L_{t}\left(\left\{x^{(n)}, y^{(n)}\right\} ; \boldsymbol{W}\right) \hat{\boldsymbol{W}}=\underset{\boldsymbol{W}}{\arg \min } \sum_{n=1}^{N} L_{t}\left(\left\{x^{(n)}, y^{(n)}\right\} ; \boldsymbol{W}\right),
$$

where $N$ is the number of training examples and $\left\{x^{(n)}, y^{(n)}\right\}$ represents $n$-th example in the training set with corresponding manual segmentation. The pixel-wise segmentation loss per image can be defined as: 
$L_{t}(\{x, y\} ; \boldsymbol{W})=-\sum_{i \in \Omega_{p} j} \sum_{j=1}^{L} y_{i}^{j} \log P\left(y_{i}^{j}=1 \mid x_{i} ; \boldsymbol{W}\right) L_{t}(\{x, y\} ; \boldsymbol{W})=-\sum_{i \in \Omega_{p} j=1}^{2} y_{i}^{j} \log P\left(y_{i}^{j}=1 \mid x_{i} ; \boldsymbol{W}\right)$,

$P\left(y_{i}^{j}=1 \mid x_{i} ; \boldsymbol{W}\right)=\frac{\exp \left(a_{j}\left(x_{i}\right)\right)}{\sum_{k=1}^{L} \exp \left(a_{k}\left(x_{i}\right)\right)} P\left(y_{i}^{j}=1 \mid x_{i} ; \boldsymbol{W}\right)=\frac{\exp \left(a_{j}\left(x_{i}\right)\right)}{\sum_{k=1}^{L} \exp \left(a_{k}\left(x_{i}\right)\right)}$,

where $a_{j}\left(x_{i}\right)$ is the output of the penultimate activation layer of the network for the pixel $x_{i^{\prime}} \Omega_{p}$ represents the pixel space and $P$ are the corresponding class probabilities.

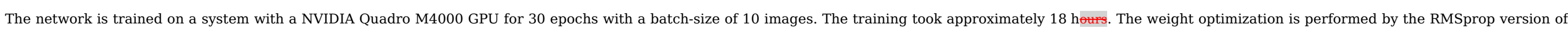
the stochastic gradient descent algorithm throughout this work [24].

\subsection{Inference and MetriesInference and metrics}

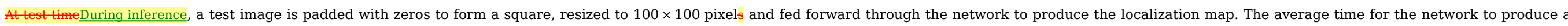

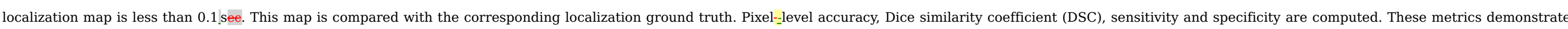

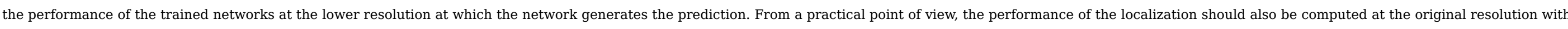

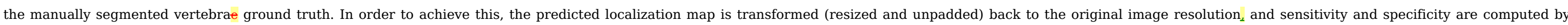
comparing them with the manually segmented vertebrae ground truth.

\subsection{Results}

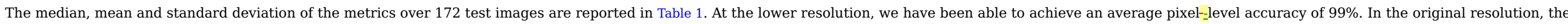

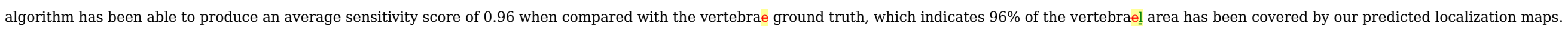

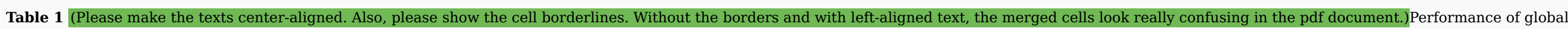
localization.

\begin{tabular}{|c|c|c|c|c|c|c|}
\hline \multirow[t]{2}{*}{ Resolution } & \multicolumn{4}{|c|}{$25 \times 25$} & \multicolumn{2}{|c|}{ Original } \\
\hline & Pixel Aạccuracy & DSC & Sensitivity & Specificity & Sensitivity & Specificity \\
\hline Median & 0.99 & 0.91 & 0.89 & 1.00 & 1.00 & 0.96 \\
\hline Mean & 0.99 & 0.89 & 0.86 & 1.00 & 0.96 & 0.96 \\
\hline Std & 0.01 & 0.10 & 0.13 & 0.00 & 0.11 & 0.01 \\
\hline
\end{tabular}

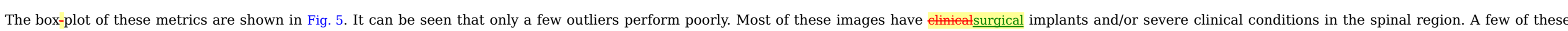

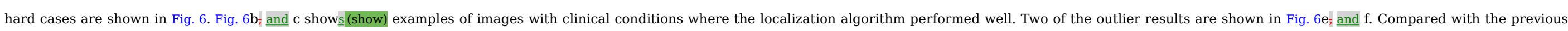

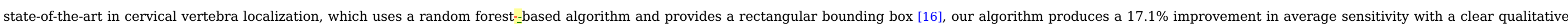

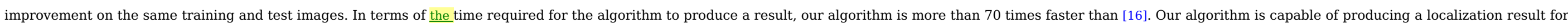
any image under a second while the sliding window-_based method of [16] requires 70te_180 seonds depending on the image size.

\section{Center LocalizationCenter localization}

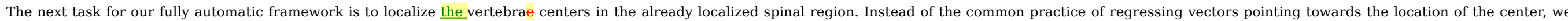




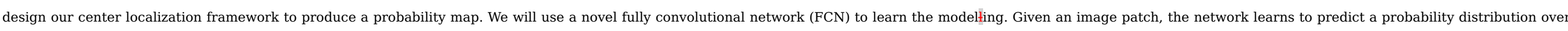
the image space indicating where the centers are most probable. In contrary to the vector regression techniques, our method can predict multiple centers for a single patch.

\subsection{Data}

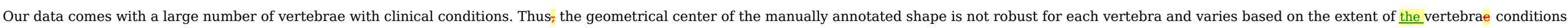

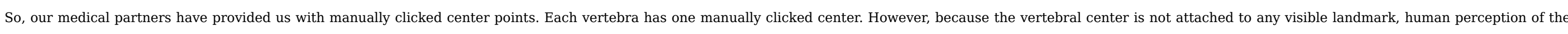
center also varies to some extent. This motivated us to convert the manually clicked centers into probabilistic distributions.

The probability distribution at a vertebral center $\left(x_{C}, y_{c}\right)$ can be defined as a 2D anisotropic Gaussian distribution [25].

\begin{tabular}{|l|l|}
\hline$F(x, y)=\frac{1}{2 \pi \sqrt{v_{w} v_{h}}} e^{-\frac{1}{2 v_{x} v_{y}}\left(a_{1}\left(x-x_{c}\right)^{2}-2 a_{2}\left(x-x_{c}\right)\left(y-y_{c}\right)+a_{3}\left(y-y_{c}\right)^{2}\right)}$ & $F(x, y)=\frac{1}{2 \pi \sqrt{v_{w} v_{h}}} e^{-\frac{1}{2 v_{w} v_{h}}\left(a_{1}\left(x-x_{c}\right)^{2}-2 a_{2}\left(x-x_{c}\right)\left(y-y_{c}\right)+a_{3}\left(y-y_{c}\right)^{2}\right)}$, \\
\hline
\end{tabular}

where

$a_{1}=v_{w} \cos ^{2} \theta+v_{h} \sin ^{2} \theta \quad a_{1}=v_{w} \cos ^{2} \theta+v_{h} \sin ^{2} \theta$,

$a_{2}=\left(v_{w}-v_{h}\right) \cos \theta \sin \theta a_{2}=\left(v_{w}-v_{h}\right) \cos \theta \sin \theta$,

$a_{3}=v_{w} \sin ^{2} \theta+v_{h} \cos ^{2} \theta a_{3}=v_{w} \sin ^{2} \theta+v_{h} \cos ^{2} \theta$,

and

$\theta=\frac{\theta_{l}+\theta_{b}+\theta_{r}+\theta_{t}}{4} \theta=\frac{\theta_{l}+\theta_{b}+\theta_{r}+\theta_{t}}{4}$,

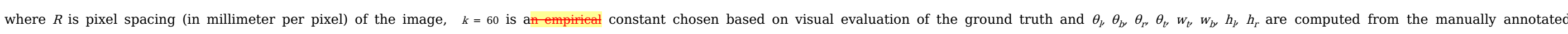
vertebrae eground truthers and demonstrated in Fig. 7a.

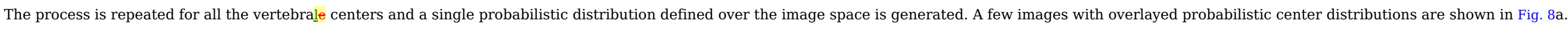

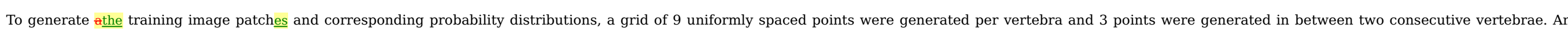

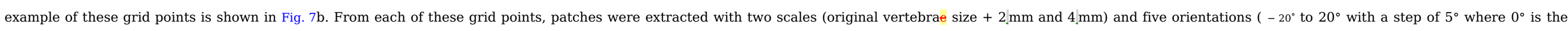

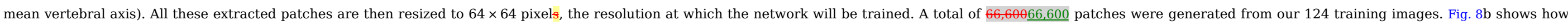
these distributions look at the patch--level.

\subsection{Network}

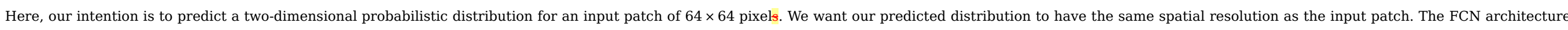

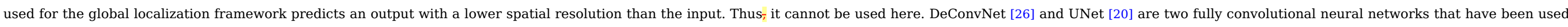

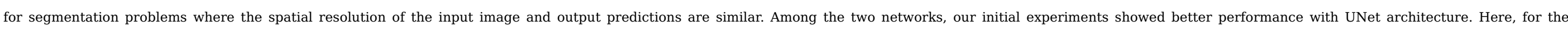

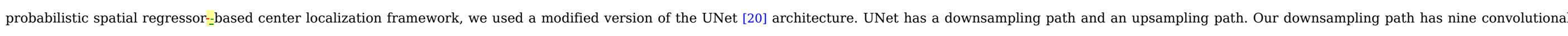

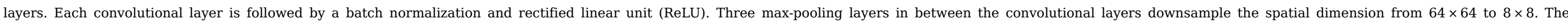




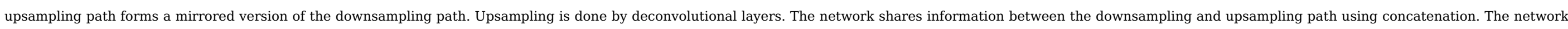
diagram is shown in Fig. 9. The number of filters in each layer can be tracked from the number of channels in the data blocks. The total number of parameters for the center localization UNet is $24,238,210$.

\subsection{Training}

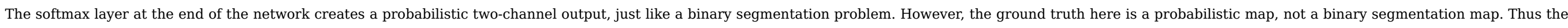
standard segmentation log loss of Section 3.3 cannot be used. We formulate a novel loss function for training the network to predict a probabilistic map.

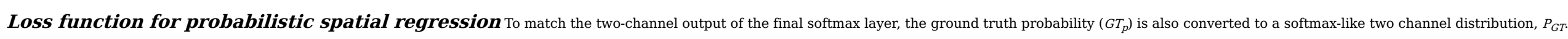

$$
\begin{aligned}
& P_{G T_{i, \text { channel }=1}}=\frac{G T_{p_{i}}-\min \left(G T_{p}\right)}{\max \left(G T_{p}\right)-\min \left(G T_{p}\right)} P_{G T_{i, \text { hannel }=1}}=\frac{G T_{p_{i}}-\min \left(G T_{p}\right)}{\max \left(G T_{p}\right)-\min \left(G T_{p}\right)},
\end{aligned}
$$

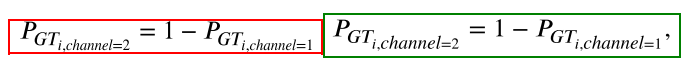

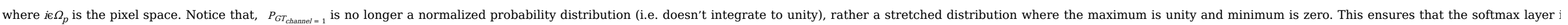
able to produce a similar distribution, as it squashes the input activations to the range from 0 to 1 .

Training our UNet would then mean finding an optimized set of parameters $\widehat{W}_{o}$ which minimizes a loss, $L$, between the predicted $\widehat{y}^{(n)}$ and updated ground truth $P_{G T}^{(n)}$ over the training dataset.

$$
\widehat{\boldsymbol{W}}_{o}=\underset{\boldsymbol{W}}{\arg \min } \sum_{n=1}^{N} L\left(\left\{x^{(n)}, P_{G T}^{(n)}\right\} ; \boldsymbol{W}\right) \widehat{W}_{o}=\underset{\boldsymbol{W}}{\arg \min } \sum_{n=1}^{N} L\left(\left\{x^{(n)}, P_{G T}^{(n)}\right\} ; \boldsymbol{W}\right),
$$

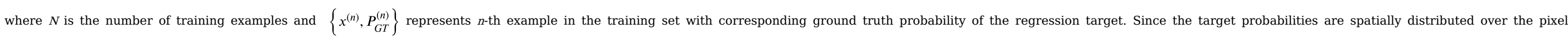
space, we can define a pixel-wise loss function per training sample as:

$$
L\left(\left\{x, P_{G T}\right\} ; \boldsymbol{W}\right)=\frac{1}{2\left|\Omega_{p}\right|} \sum_{i \in \Omega_{p}} \sum_{j=1}^{2} w_{i}\left(\hat{y}_{i}-P_{G T_{i, c h a n n e l=j}}\right)^{2} L\left(\left\{x, P_{G T}\right\} ; \boldsymbol{W}\right)=\frac{1}{2\left|\Omega_{p}\right|} \sum_{i \in \Omega_{p}} \sum_{j=1}^{2} w_{i}\left(\hat{y}_{i}-P_{G T_{i, c h a n n e l=j}}\right)^{2},
$$

where

$w_{i}=\left\{\begin{array}{cc}\frac{\left|\Omega_{p_{\phi}}\right|}{\left|\Omega_{p_{o}}\right|} & \text { if } i \epsilon \Omega_{p_{\phi}} \\ 1 & \text { otherwise }\end{array} w_{i}=\left\{\begin{array}{cc}\frac{\left|\Omega_{p_{\phi}}\right|}{\left|\Omega_{p_{o}}\right|} & \text { if } i \epsilon \Omega_{p_{\phi}} \\ 1 & \text { otherwise }\end{array}\right.\right.$

where $\Omega_{p}$ is the pixel space, $\Omega_{p_{\phi}}$ is set of pixels where the ground truth probabilities are not zero and $\Omega_{p_{o}}=\Omega_{p}-\Omega_{p_{\phi}}$.

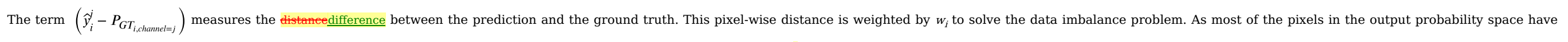

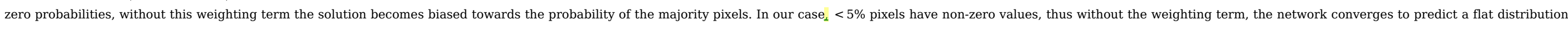
of zeros.

The network is trained on a system with a NVIDIA Pascal Titan X GPU for 30 epochs with a batch-size of 25 image patches. The training took approximately 72 hours.

\subsection{Inference and Post-processing Inference and post-processing}

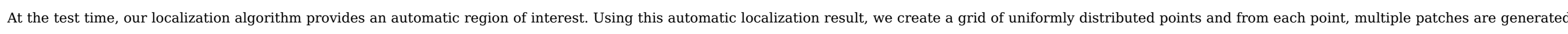

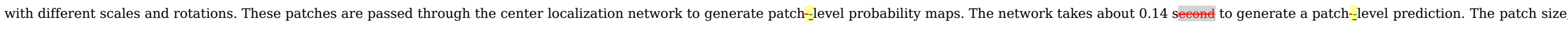

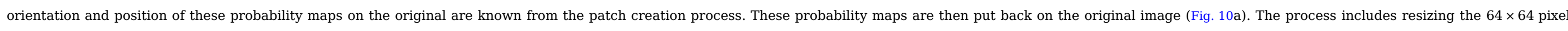

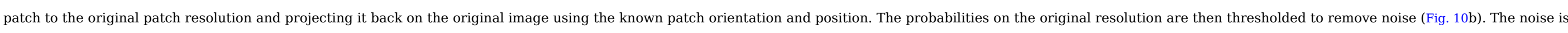




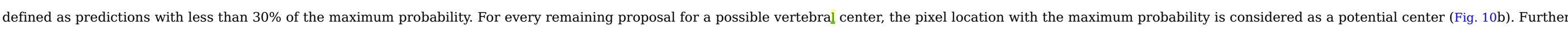

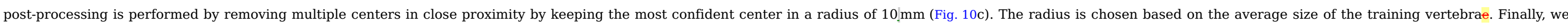
keep the maximum number of possible centers to five (C3-C7) and delete less confident center proposals if more than five centers are detected (Fig. 10d).

\subsection{Experiments and MetriesExperiments and metrics}

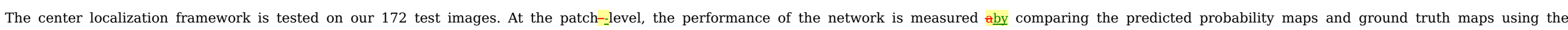
Bhattacharyya coefficient [27].

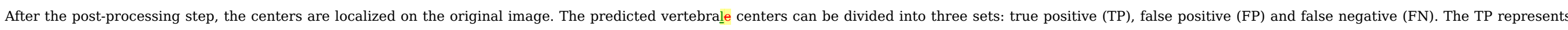

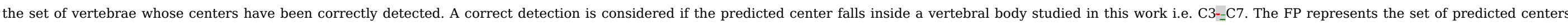

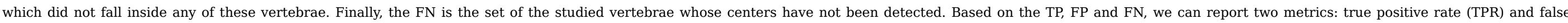
discovery rate (FDR) [28]. We also report the Euclidean distance between the correctly detected centers and corresponding ground truth in mm as the distance error

$T P R=\frac{|T P|}{|T P|+|F N|} \times 100 \% \quad T P R=\frac{|T P|}{|T P|+|F N|} \times 100 \%$.
$F D R=\frac{|F P|}{|F P|+|T P|} \times 100 \% \quad F D R=\frac{|F P|}{|F P|+|T P|} \times 100 \%$.

\subsection{Results}

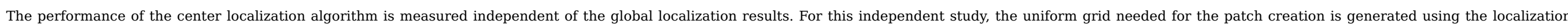

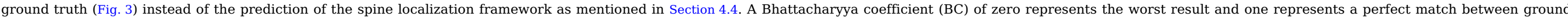

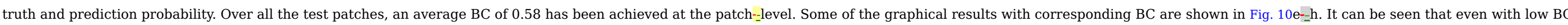

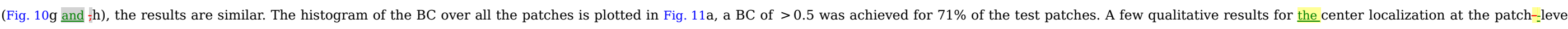
are shown in Fig. 11b.

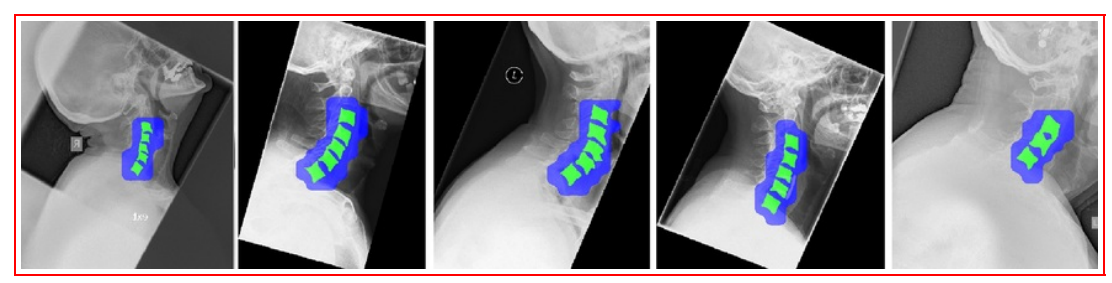

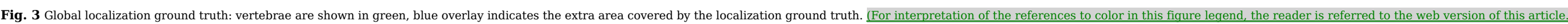
alt-text: Fig. 3

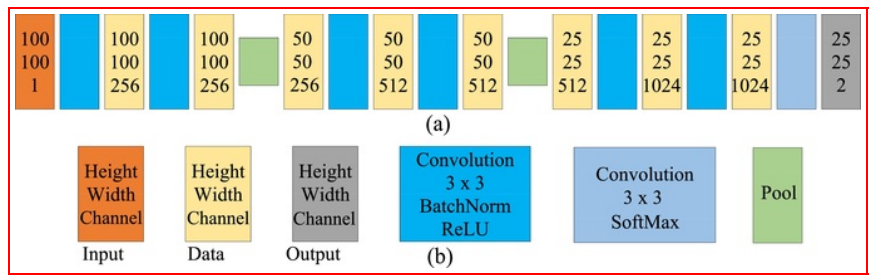

Fig. 4 Fully convolutional network for localization of spinal region (a) Network architecture! (b) Legends.

alt-text: Fig. 4 


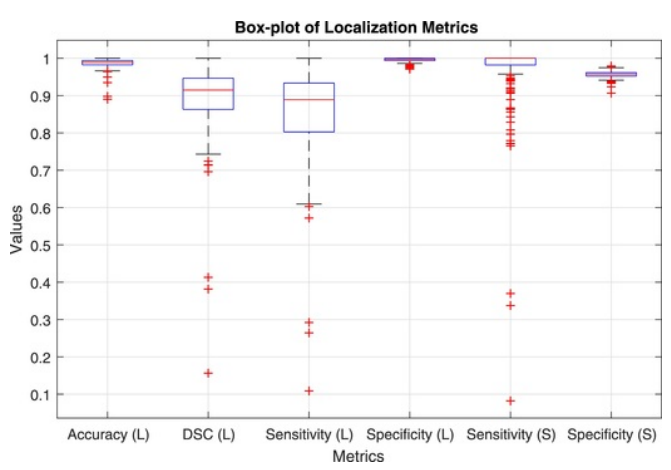

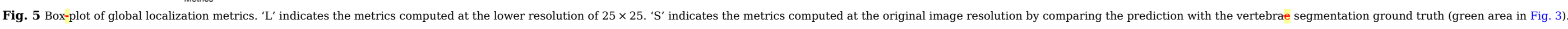
(For interpretation of the references to color in this figure legend, the reader is referred to the web version of this article.

\section{alt-text: Fig. 5}

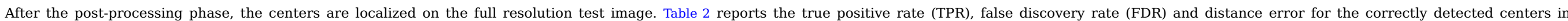
millimeters $(\mathrm{mm})$

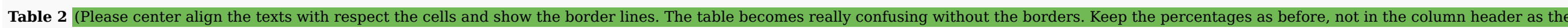

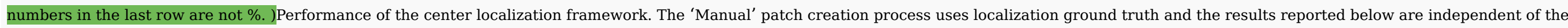

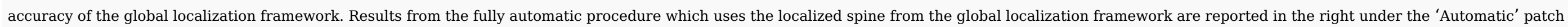
creation process.

\section{alt-text: Table 2}

True positive rate (TPR)

False discovery rate (FDR)

Distance error (mm)
Test patch creation

$93.73 \%$

$4.72 \%$

Median

1.63
Manual (\%)

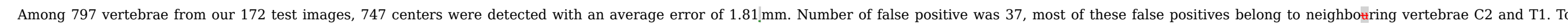

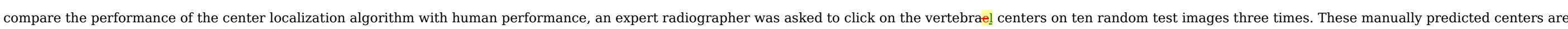

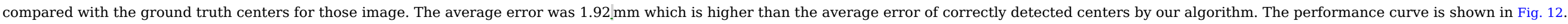

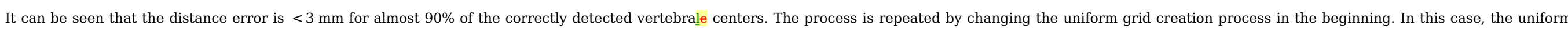

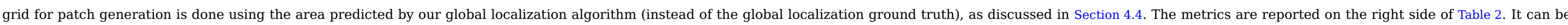

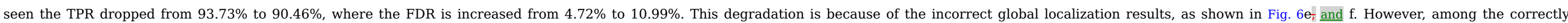

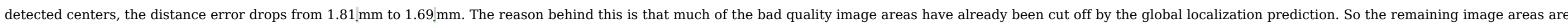
of comparatively of good quality thus the center localization performs better on average on these image areas. Some graphical center localization results in the original resolution are shown in Fig. 13 . 


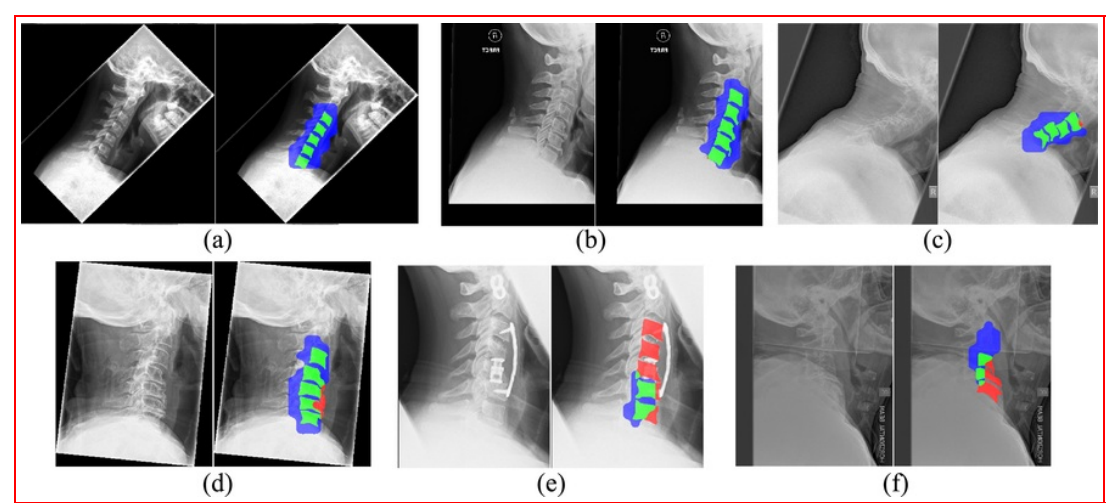

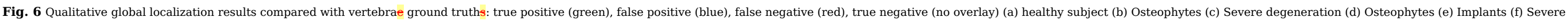
degeneration and osteophytes. (For interpretation of the references to color in this fiqure lecend, the reader is referred to the web version of this article.) alt-text: Fig. 6

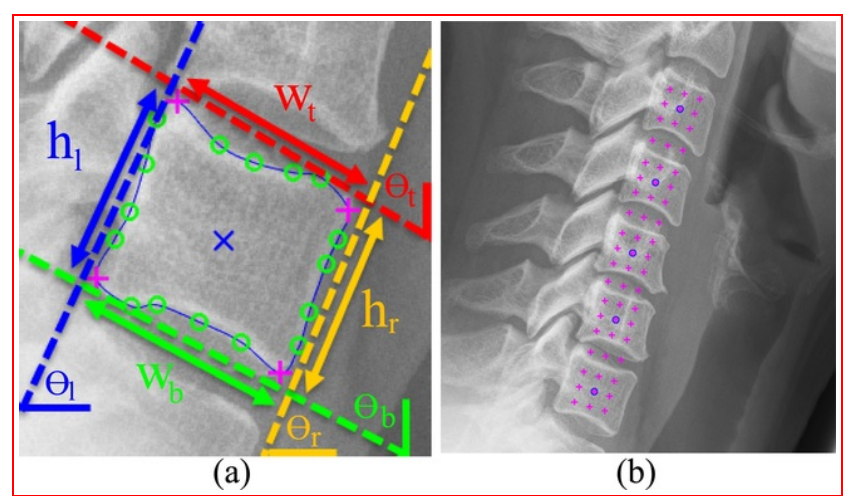

Fig. 7 (a) Probabilistic ground truth creation: manually clicked vertebral center $(X)$, manually annotated vertebral boundary $(O)$ and corner $(+)$ points (b) Grid points $(+)$ for training patches. alt-text: Fig. 7

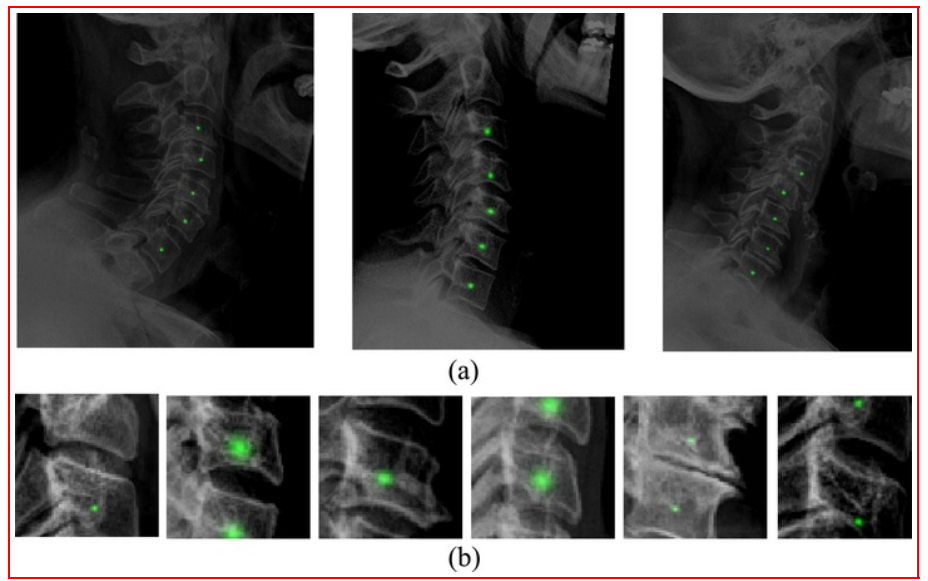




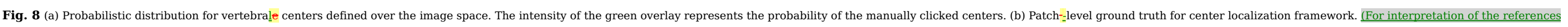
to color in this figure legend, the reader is referred to the web version of this article.) alt-text: Fig. 8

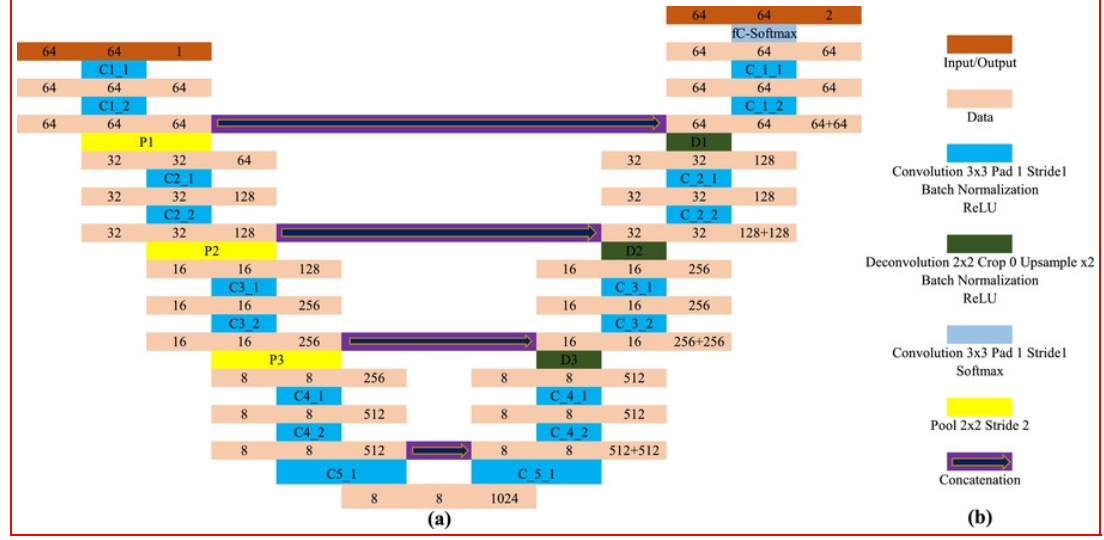

Fig. 9 UNet architecture: (a) Network diagram. (b) Legends.

alt-text: Fig. 9

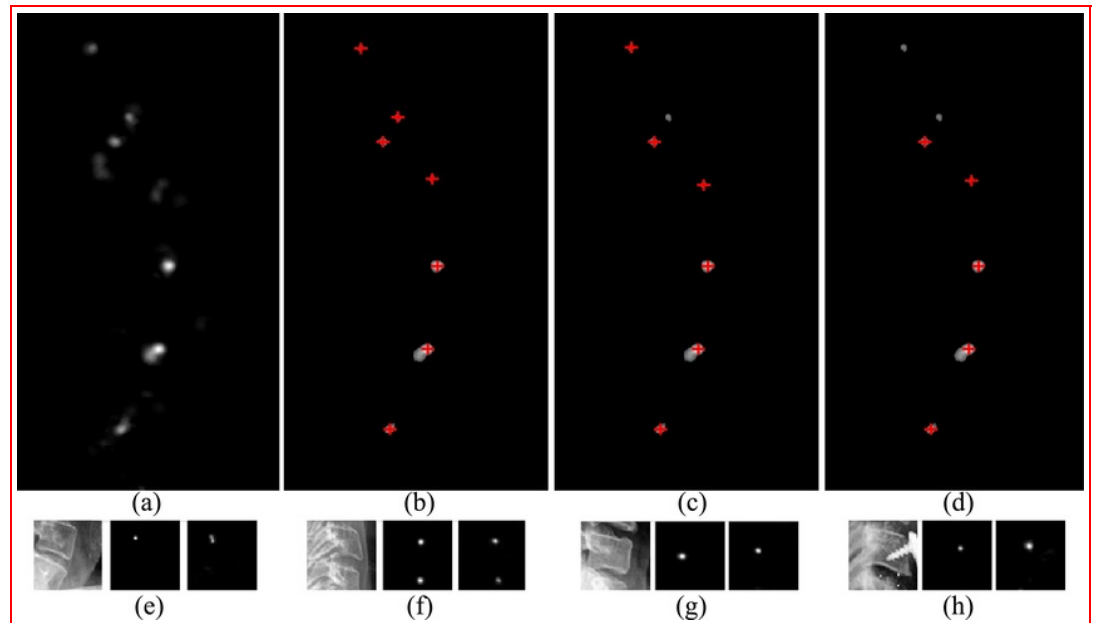

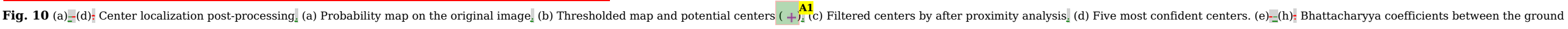

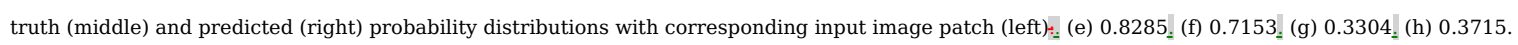

alt-text: Fig. 10

\section{Annotations:}

A1. it looks magenta, if so, please change it to red. 


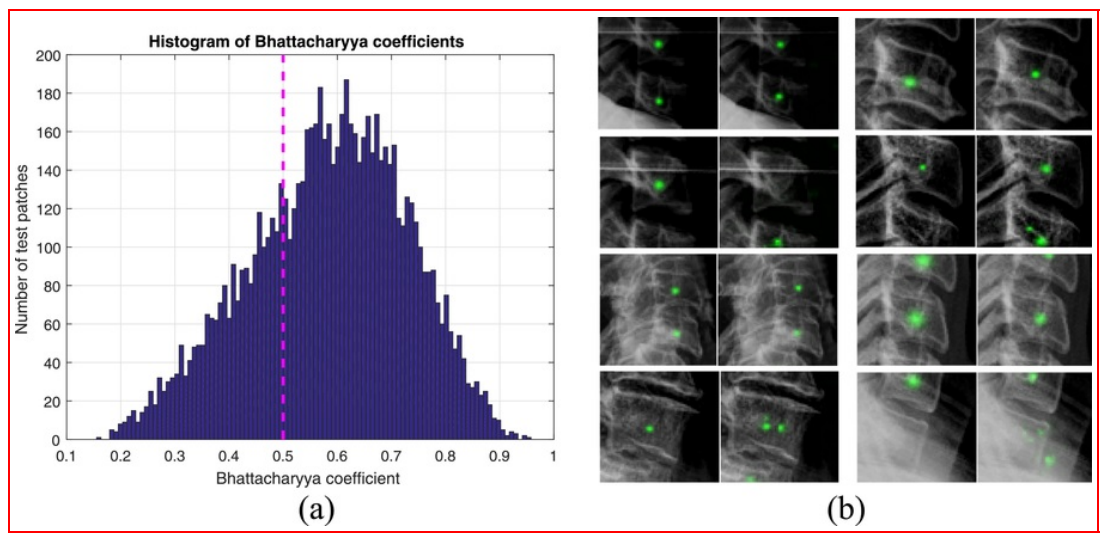

Fig. 11 (a) Histogram of Bhattacharyya coefficients!: (b) Patch-level center localization results: Gground truth (left) and Đ̣prediction (right) alt-text: Fig. 11

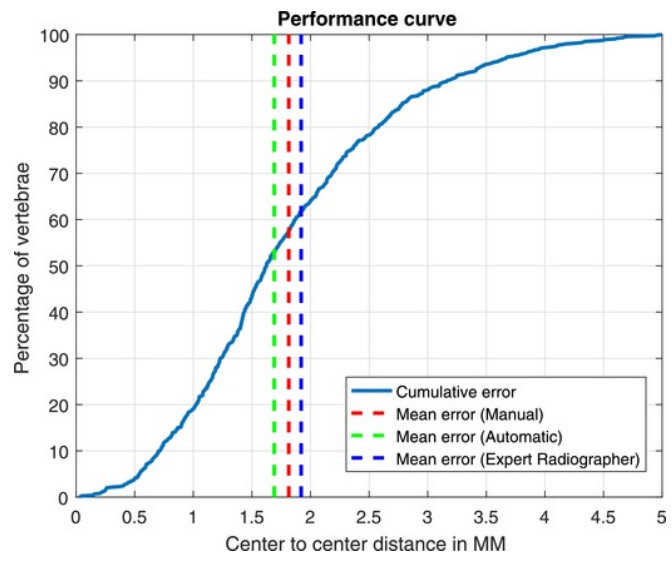

Fig. 12 Performance curve for center localization.

alt-text: Fig. 12 


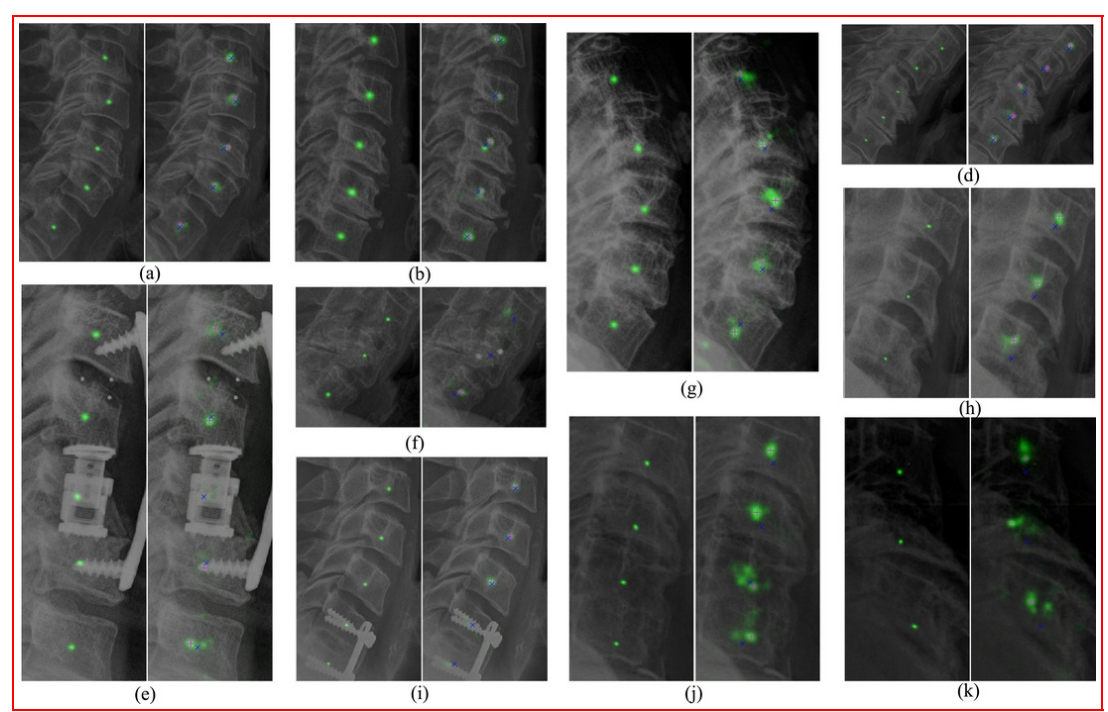

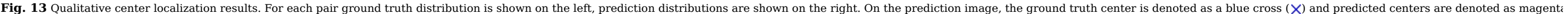
plus $(+)$. (For interpretation of the references to color in this fiqure lecend, the reader is referred to the web version of this article.) alt-text: Fig. 13

\section{Vertebrae SegmentationVertebrae segmentation}

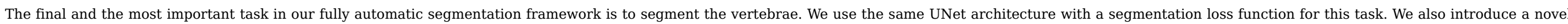
shape-aware term in segmentation loss function to predict the vertebrale shape with better accuracy.

\subsection{Data}

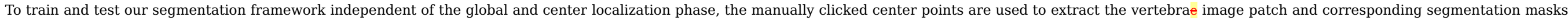

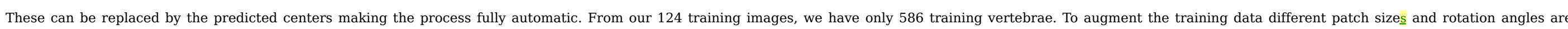

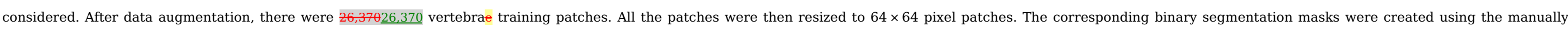

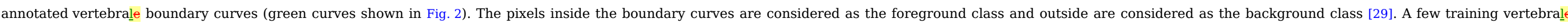

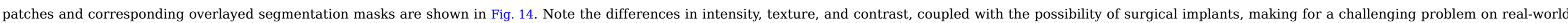
data. Similarly, vertebrale patches were also collected from the test images, a total of 797 vertebrae were extracted. No augmentation was performed for the test vertebrae.

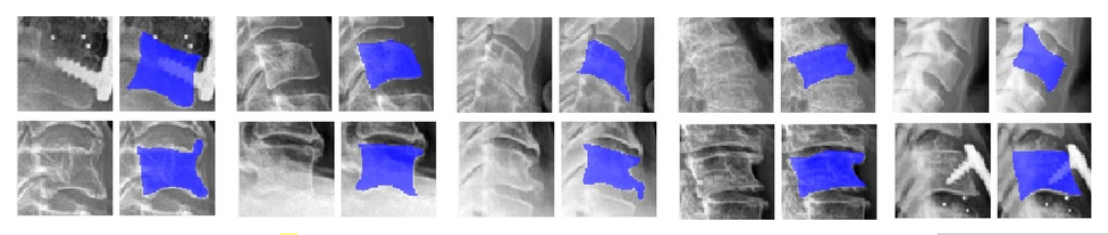

Fig. 14 Training vertebrale patches and corresponding segmentation masks (blue overlay). (For interpretation of the references to color in this figure legend, the reader is referred to the web version of this article.) alt-text: Fig. 14

\subsection{Training}




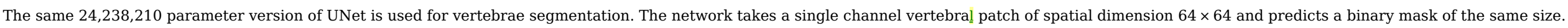

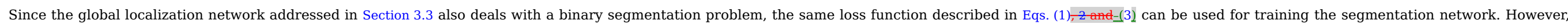

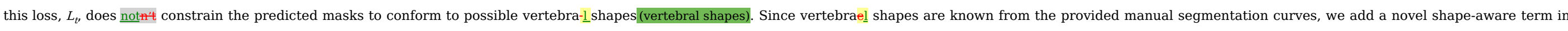
the loss function to force the network to learn to penalize predicted areas outside the curve.

\subsection{Shape-aware Loss TermShape-aware loss term}

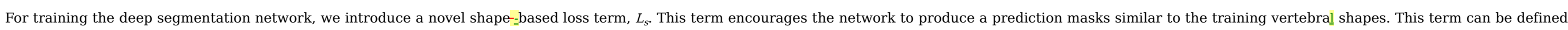
as:

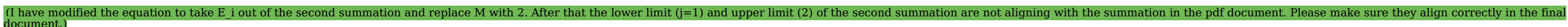
$L_{s}(\{x, y\} ; \boldsymbol{W})=-\sum_{i \in \hat{\Omega}_{p}} \sum_{j=1}^{M} y_{i}^{j} E_{i} \log P\left(y_{i}^{j}=1 \mid x_{i} ; \boldsymbol{W}\right) L_{s}(\{x, y\} ; \boldsymbol{W})=-\sum_{i \in \hat{\Omega}_{p}} E_{i} \sum_{j=1}^{2} y_{i}^{j} \log P\left(y_{i}^{j}=1 \mid x_{i} ; \boldsymbol{W}\right)$, $E_{i}=D\left(\widehat{C}, C_{G T}\right) E_{i}=D\left(\widehat{C}, C_{G T}\right)$,

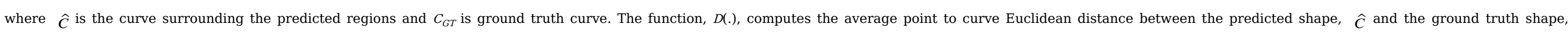

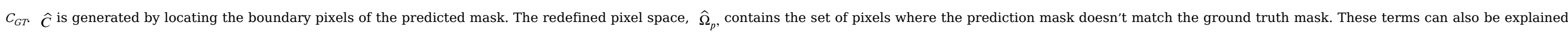

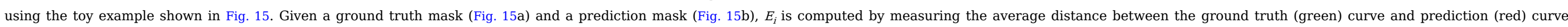

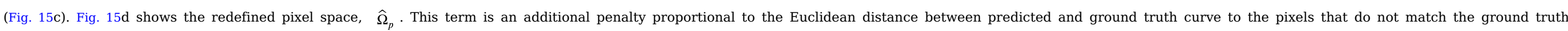

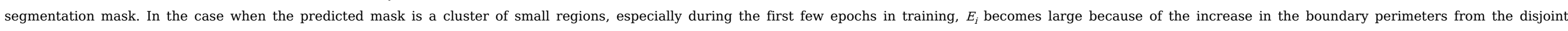
predictions.

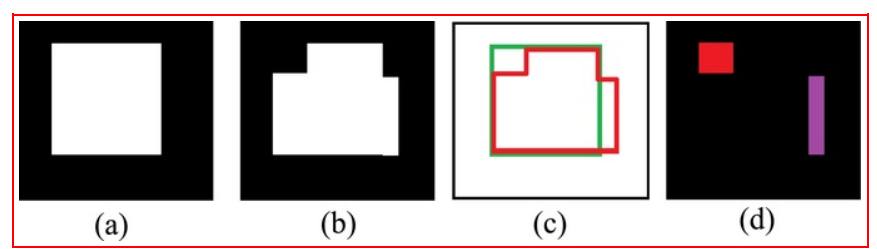

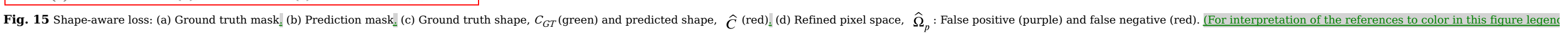
the reader is referred to the web version of this article.)

alt-text: Fig. 15

Finally, the loss function of Eq. (1) can be extended as:

$\widehat{\boldsymbol{W}}=\underset{\boldsymbol{W}}{\arg \min } \sum_{n=1}^{N}\left(L_{t}\left(\left\{x^{(n)}, y^{(n)}\right\} ; \boldsymbol{W}\right)+L_{s}\left(\left\{x^{(n)}, y^{(n)}\right\} ; \boldsymbol{W}\right)\right) \hat{\boldsymbol{W}}=\underset{\boldsymbol{W}}{\arg \min } \sum_{n=1}^{N}\left(L_{t}\left(\left\{x^{(n)}, y^{(n)}\right\} ; \boldsymbol{W}\right)+L_{s}\left(\left\{x^{(n)}, y^{(n)}\right\} ; \boldsymbol{W}\right)\right)$.

The contribution of each term in the total loss can be controlled by introducing a weight parameter in Eq. (17). However, in our case, the best performance was achieved when both terms contributed equally.

\subsection{Experiments and MetriesExperiments and metrics}

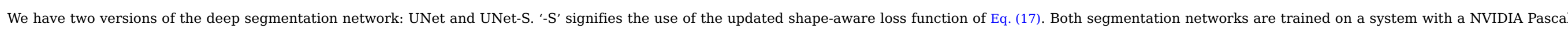

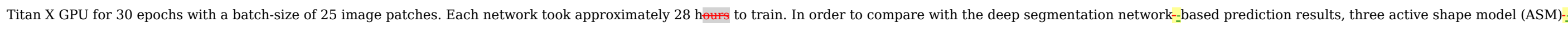

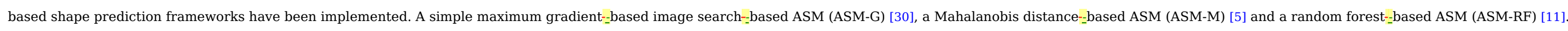




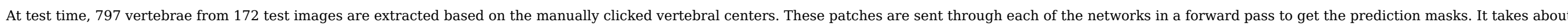

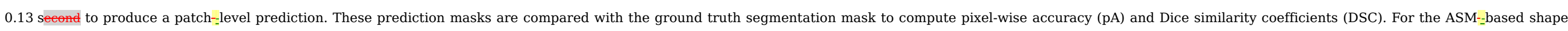

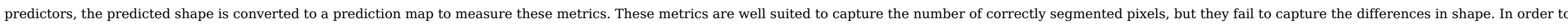

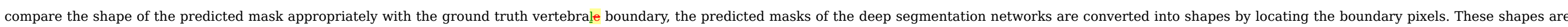

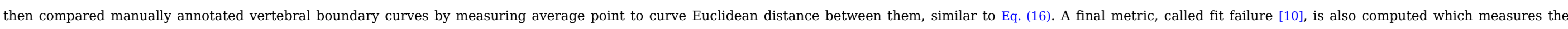
percentage of vertebrae having an average point to ground truth curve error of greater than 1_mm.

\subsection{Results}

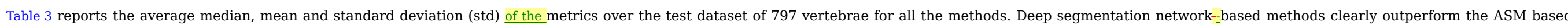

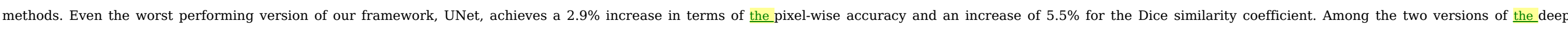

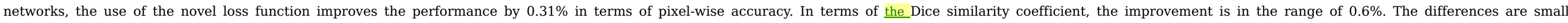

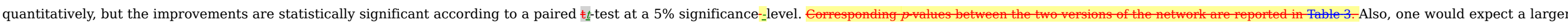

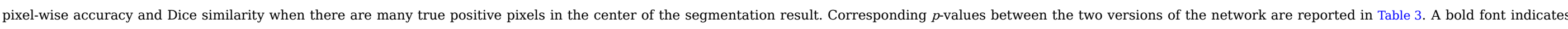

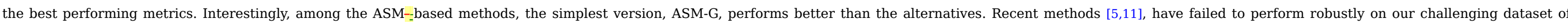
the test vertebrae.

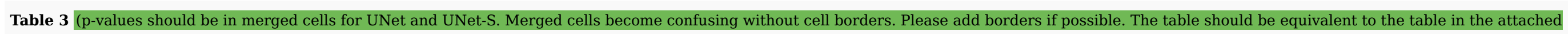
image. Also if possible, please center-align the texts both vertically and horizontally.)Average quantitative metrics for vertebrae segmentation.

alt-text: Table 3

\begin{tabular}{|c|c|c|c|c|c|c|c|c|}
\hline & \multicolumn{4}{|c|}{ Pixel-wise accuracy (\%) } & \multicolumn{4}{|c|}{ Dice similarity coefficient } \\
\hline & Median & Mean & Std & $p$-value & Median & Mean & Std & $p$-value \\
\hline ASM-RF & 95.09 & 90.77 & 8.98 & & 0.881 & 0.774 & 0.220 & \\
\hline ASM-M & 95.09 & 93.48 & 4.92 & & 0.900 & 0.877 & 0.073 & \\
\hline ASM-G & 95.34 & 93.75 & 4.48 & & 0.906 & 0.883 & 0.066 & \\
\hline UNet & 97.71 & 96.69 & 3.04 & $<10^{-12}$ & 0.952 & 0.938 & 0.048 & $<10^{-12}$ \\
\hline UNet-S & 97.92 & 97.01 & 2.79 & & 0.957 & 0.944 & 0.044 & \\
\hline
\end{tabular}

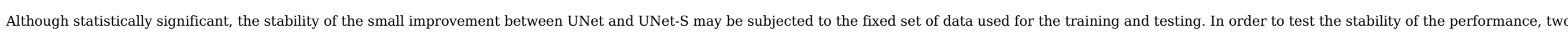

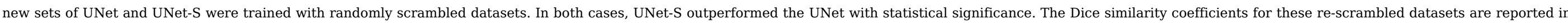
Table 4 .

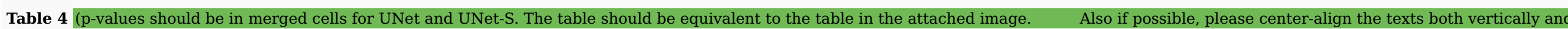
horizontally.)Dice similarity coefficients for re-scrambled datasets. 


\begin{tabular}{|c|c|c|c|c|c|c|}
\hline & Mean & Std & $p$-value & Mean & Std & $p$-value \\
\hline UNet & 0.9371 & 0.0412 & $<10^{-03}$ & 0.9433 & 0.0712 & $<.013$ \\
\hline UNet-S & 0.9411 & 0.0366 & & 0.945 & 0.0692 & \\
\hline
\end{tabular}

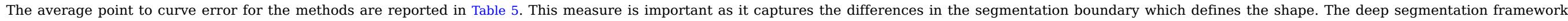

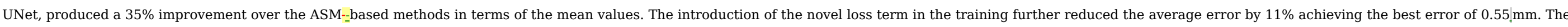

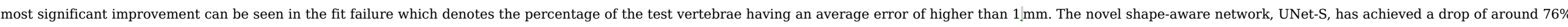

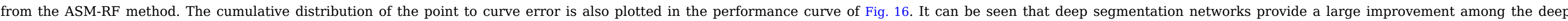
networks, shape-aware UNet performs better.

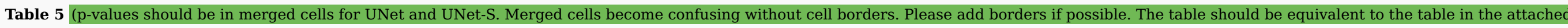
image. $\quad$ Also if possible, please center-align the texts both vertically and horizontally.)Average quantitative metric for shape prediction.

alt-text: Table 5

\begin{tabular}{|c|c|c|c|c|c|}
\hline & \multicolumn{4}{|c|}{ Average point to curve error in $\mathrm{mm}$} & \multirow[t]{2}{*}{ Fit failure(\%) } \\
\hline & Median & Mean & Std & $p \underline{p}$-value & \\
\hline ASM-RF & 1.51 & 1.74 & 0.95 & & 74.40 \\
\hline ASM-M & 0.87 & 1.02 & 0.56 & & 39.52 \\
\hline ASM-G & 0.77 & 0.95 & 0.54 & & 31.49 \\
\hline UNet & 0.43 & 0.62 & 0.81 & $\theta .0062$ & 9.41 \\
\hline UNet-S & 0.44 & 0.55 & 0.40 & & 7.40 \\
\hline
\end{tabular}

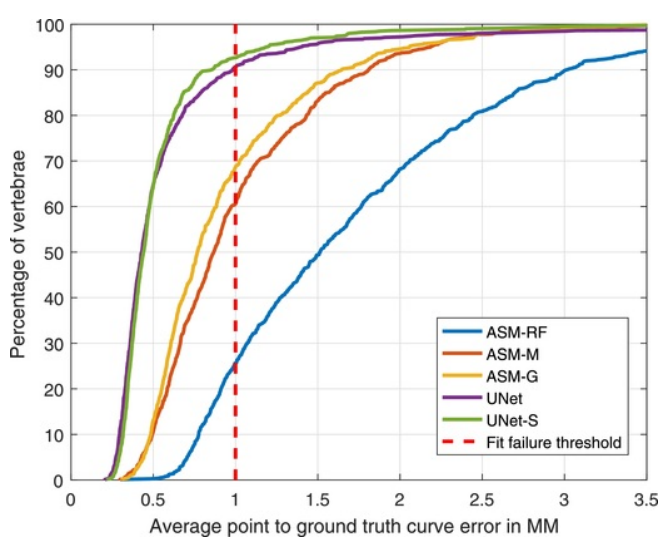

Fig. 16 Performance curve: Ęcumulative distribution of point to curve errors. alt-text: Fig. 16

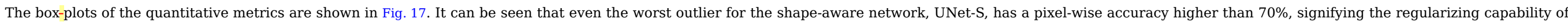

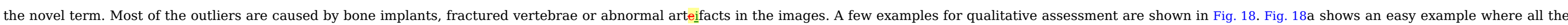




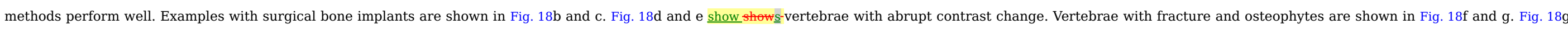

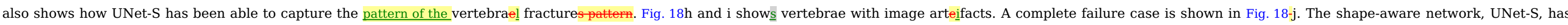
produced better segmentation results than its counterpart, UNet. Qualitatively we conclude that the novel shape-aware term provides equivalent or improved results in nearly all cases.

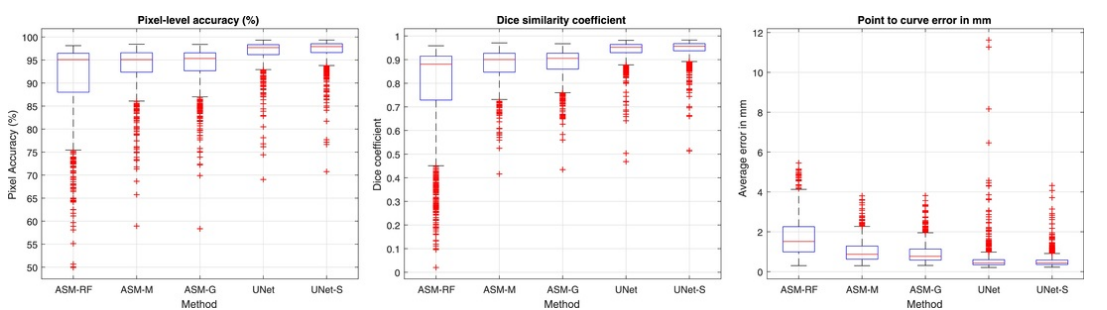

Fig. 17 Box-plots of the quantitative metrics: pixel-level accuracy (left), Dice similarity coefficients (middle) and point to manual segmentation curve error (right).

alt-text: Fig. 17 


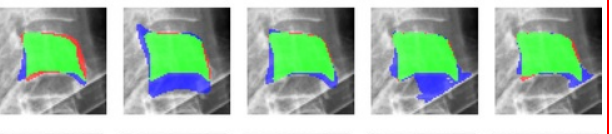

\section{alt-text: Fig. 18}

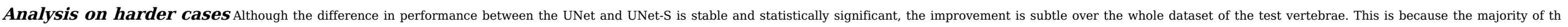

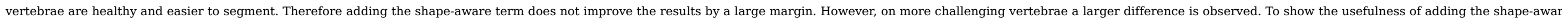

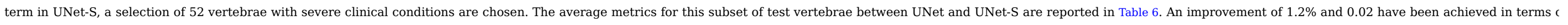

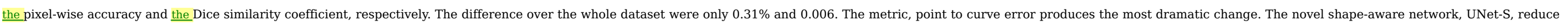
the error by $25 \%$ for this subset of vertebrae with severe clinical conditions. Fig. 19 shows a few examples of these images.

Table 6 Comparison of UNet and UNet-S for vertebrae with clinical conditions. 


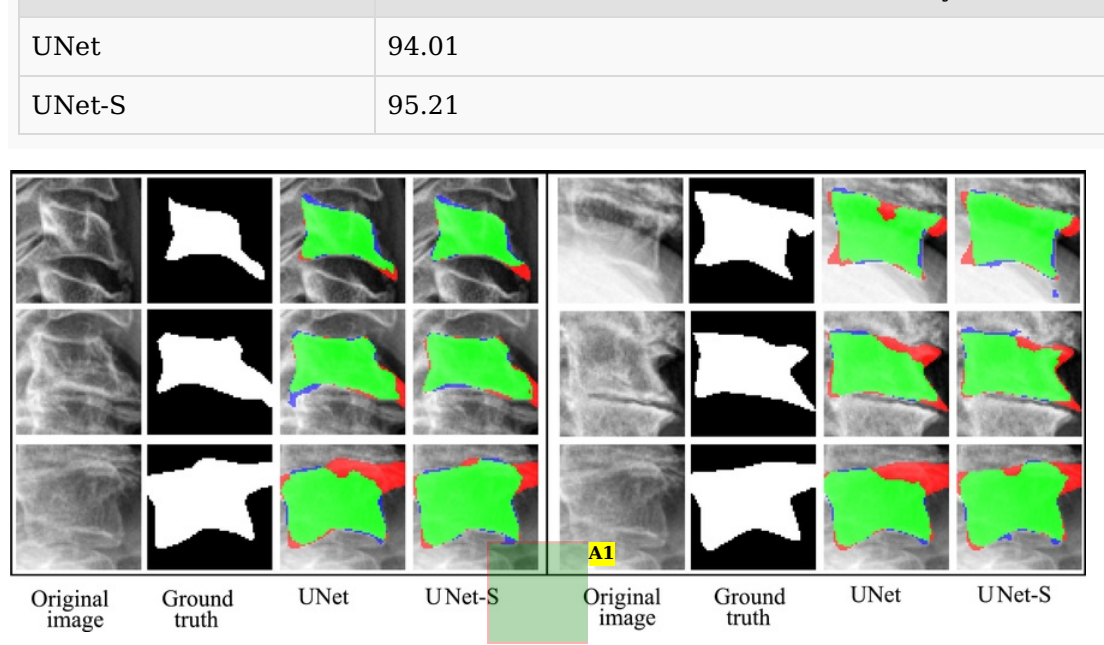

Fig. 19 Comparison of performance for vertebrae with severe clinical condition.

alt-text: Fig. 19

Annotations:

A1. will it be possible to replace the embedded text in this figure with normal text as provided in the original pdf version of this image? if not possible to put text accordingly please leave the figure as it is.

\section{Fully Automatic Segmentation FrameworkFully automatic segmentation framework}

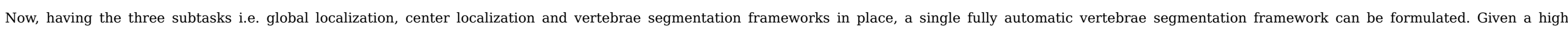

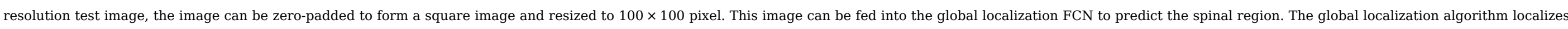
the spinal region at a lower resolution of $25 \times 25$ pixel, which can then be transformed back, i.e. resizing and unpadding, to the original image. The process is summarized in Fig. 20-1.

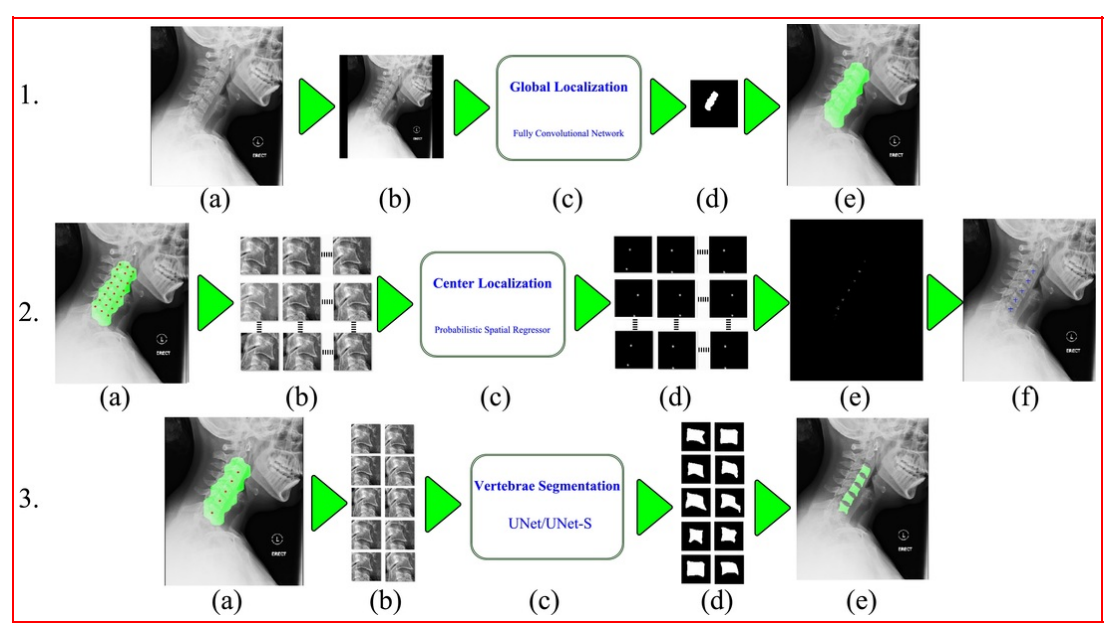

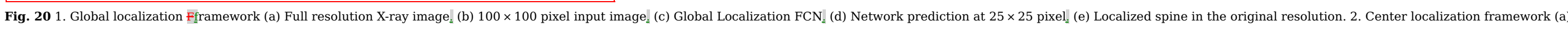

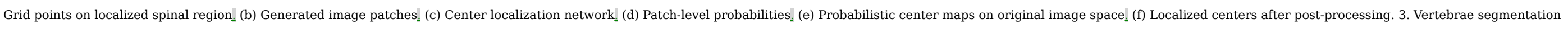




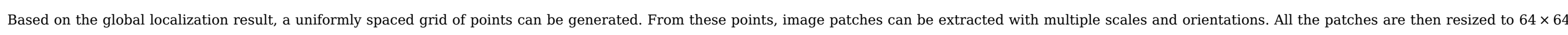

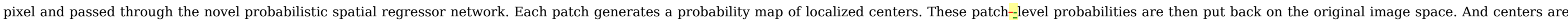
localized using the post-processing steps of Section 4.4. Fig. 20-2 depicts the center localization process.

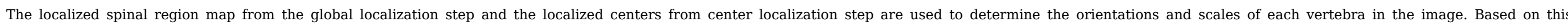

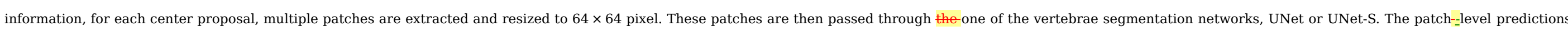
are then put back on the original image space to create the final segmentation results. The process of the vertebrae segmentation is shown in Fig. 20-3.

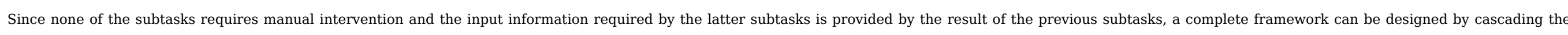

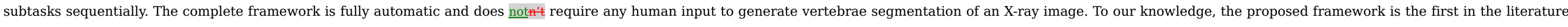

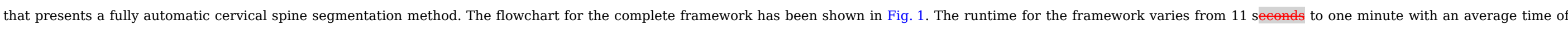

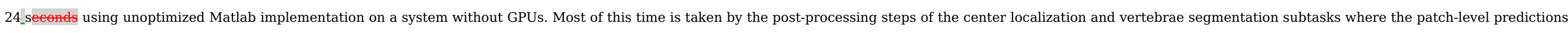
are transformed back to the original image space.

\subsection{Results}

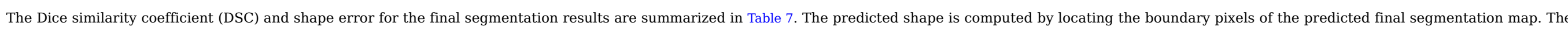

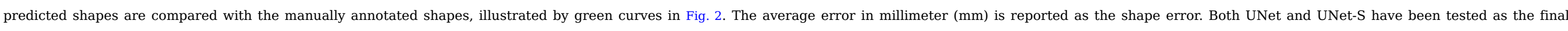

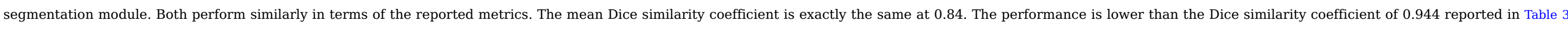

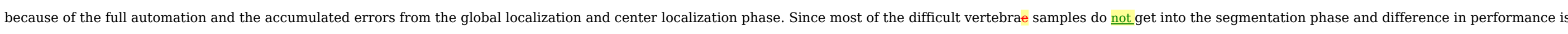

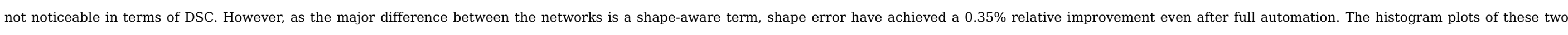
metrics are shown in Fig. 21.

Table 7 Performance of fully automatic framework.

\section{alt-text: Table 7}

\begin{tabular}{|c|c|c|c|c|}
\hline & \multicolumn{2}{|c|}{ Dice similarity coefficients } & \multicolumn{2}{|c|}{ Shape error in mm } \\
\hline & Mean & Std & Mean & Std \\
\hline UNet & 0.840 & 0.136 & 1.695 & 2.614 \\
\hline UNet-S & 0.840 & 0.135 & 1.689 & 2.555 \\
\hline
\end{tabular}

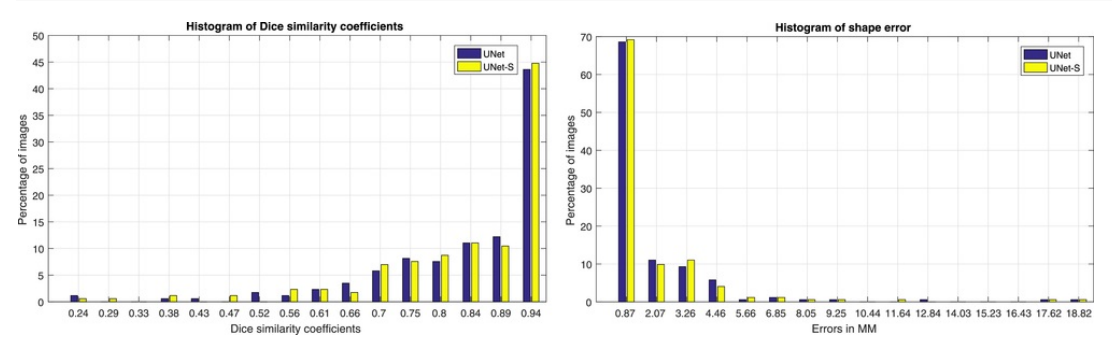

Fig. 21 Histogram plot of Dice similarity coefficients (tepleft) and shape error (nully automatic framework with UNet and UNet-S. 


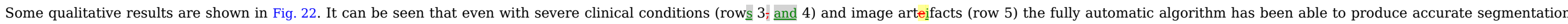

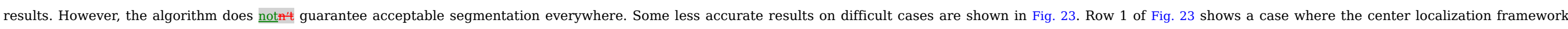

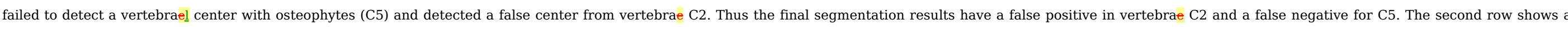

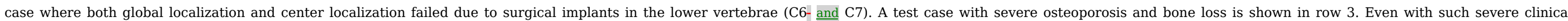

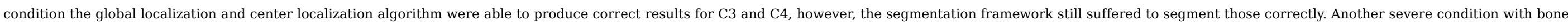

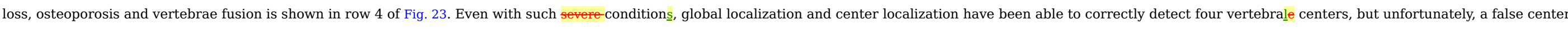

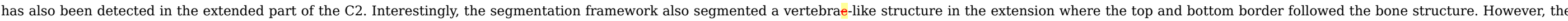

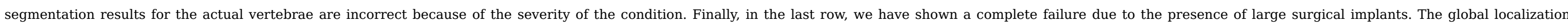
algorithm failed completely thus the following subtasks were not able to perform either. 


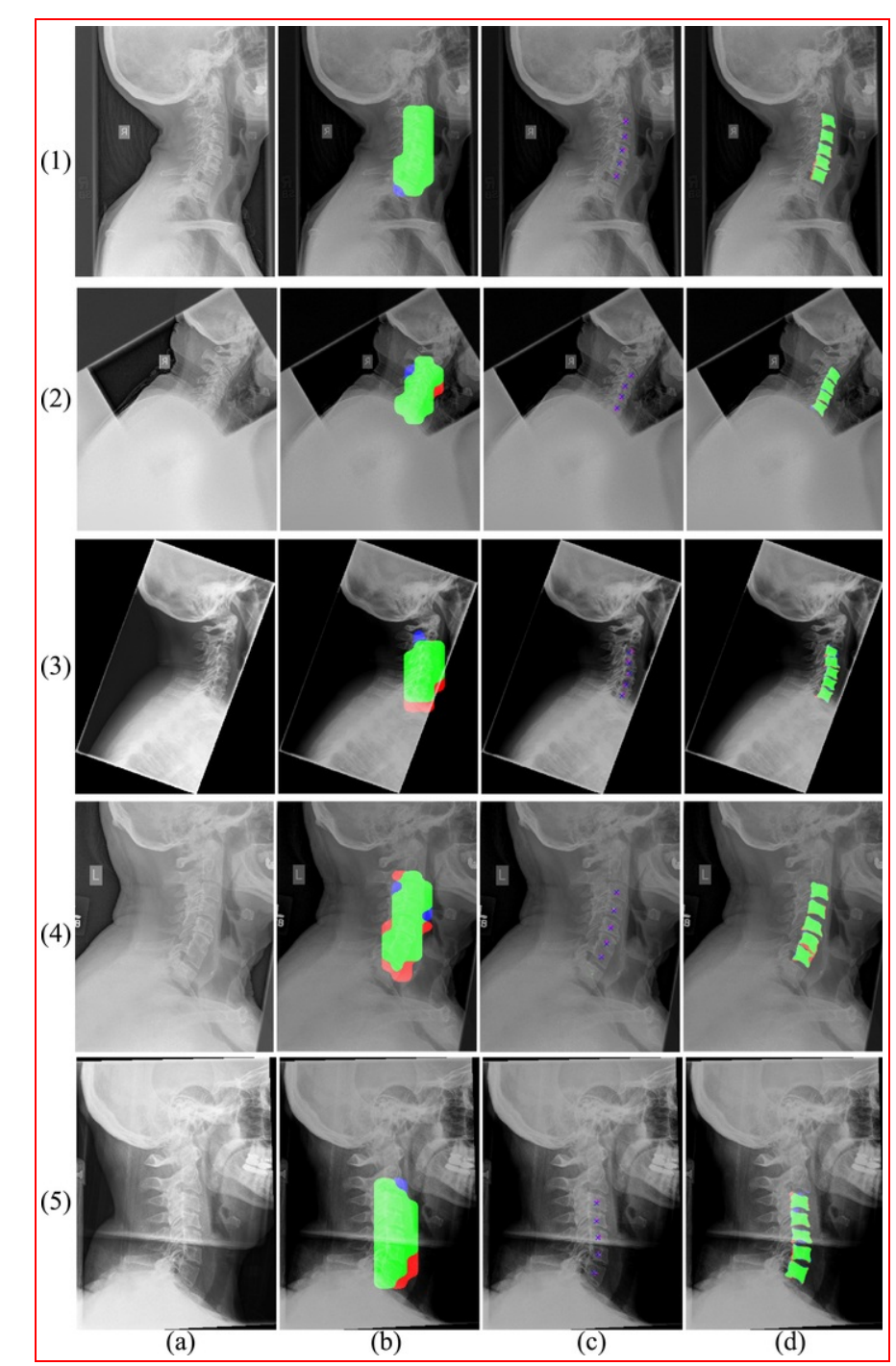

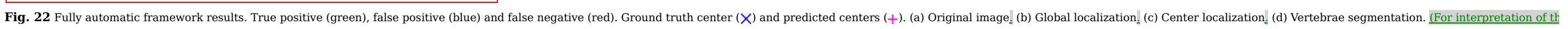
references to color in this figure legend, the reader is referred to the web version of this article.) alt-text: Fig. 22 


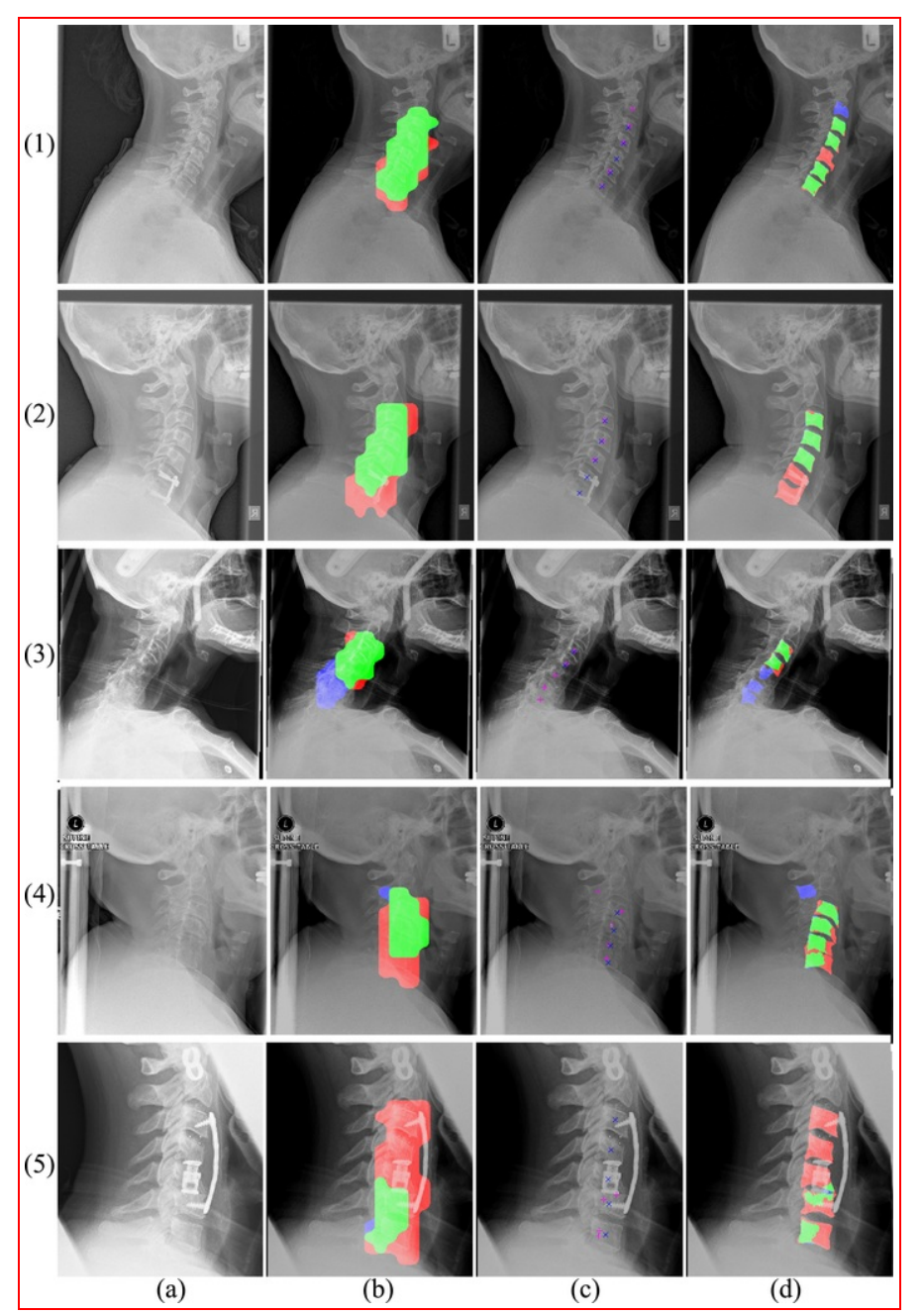

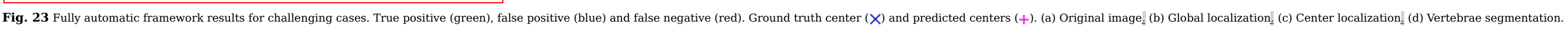
(For interpretation of the references to color in this figure lecend, the reader is referred to the web version of this article.) alt-text: Fig. 23

\section{Conclusion}

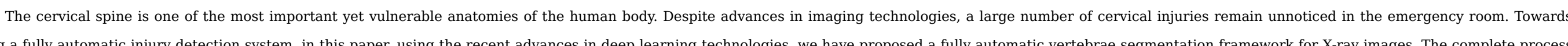

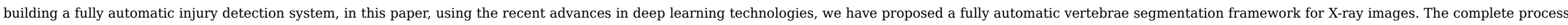

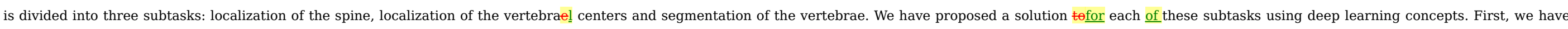

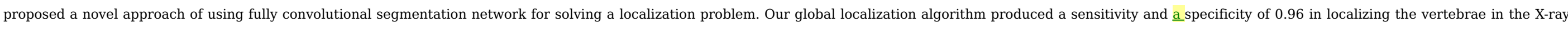

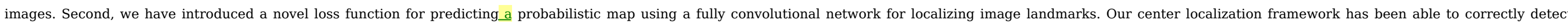

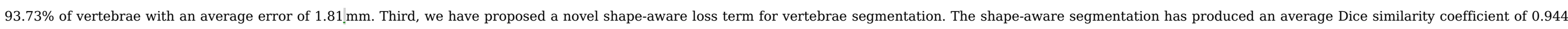




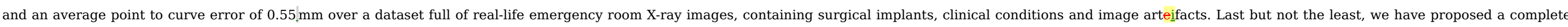
and fully automatic framework for vertebrae segmentation in X-ray images which has been able to produce a final Dice similarity coefficient of 0.84 .

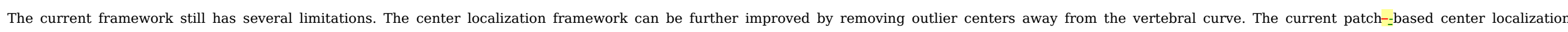

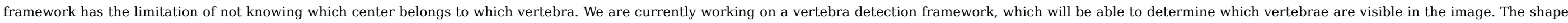

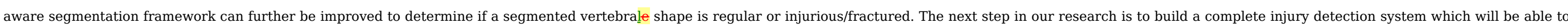

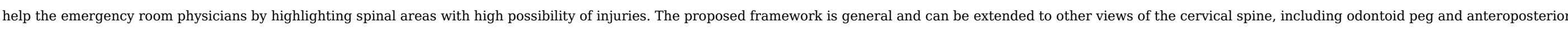
(AP) views.

\section{Acknowledgments}

We gratefully acknowledge the support of NVIDIA Corporation with the donation of the Titan X Pascal GPU used for this research.

\section{References}

[1] D. A. P. Singh, Range of Motion of Cervical Spine, (http://boneandspine.com/range-motion-cervical-spine/), Accessed: 2017-11-09.

[2] A. Singh, L. Tetreault, S. Kalsi-Ryan, A. Nouri and M.G. Fehlings, Global prevalence and incidence of traumatic spinal cord injury, foumal of Clinical Epidemiology I. Clin. Epidemiol. 6, 2014 , 309-331.

[3] P. Platzer, N. Hauswirth, M. Jaindl, S. Chatwani, V. Vecsei and C. Gaebler, Delayed or missed diagnosis of cervical spine injuries, J. Trauma Acute Care Surg. 61 (1), $2006,150-155$.

[4] C. Morris and E. McCoy, Clearing the cervical spine in unconscious polytrauma victims, balancing risks and effective screening, Anaesthesia 59 (5), 2004 , 464-482.

[5] M. Benjelloun, S. Mahmoudi and F. Lecron, A framework of vertebra segmentation using the active shape model-based approach, J. Biomed. Imaging 2011, 2011, 9.

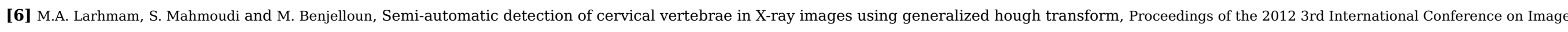
Processing Theory, Tools and Applications (IPTA), 2012, IEEE, 396-401.

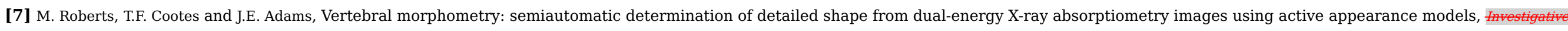
Radiology Investig. Radiol. 41 (12), 2006, 849-859.

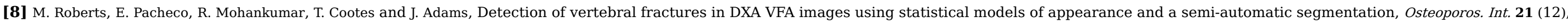
2010, 2037-2046.

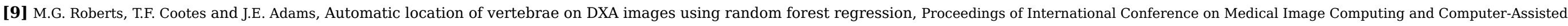
Intervention (MICCAI 2012), 2012, Springer, 361-368.

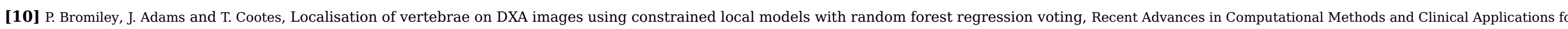
Spine Imaging, 2015, Springer, 159-171.

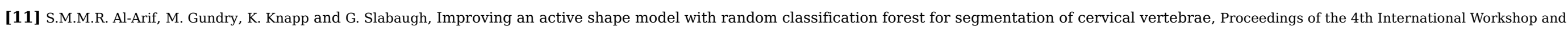

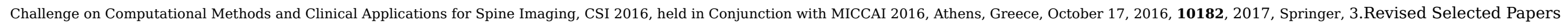

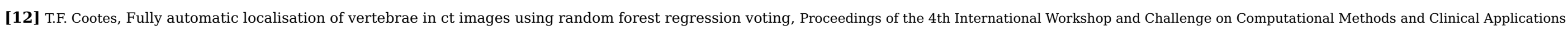
for Spine Imaging, CSI 2016, held in Conjunction with MICCAI 2016, Athens, Greece, October 17, 2016, 10182, 2017, Springer, 51.Revised Selected Papers

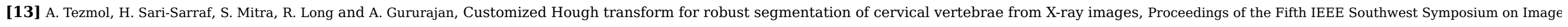
Analysis and Interpretation, 2002, 2002, IEEE, 224-228.

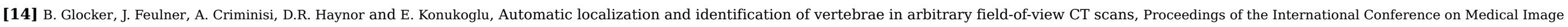




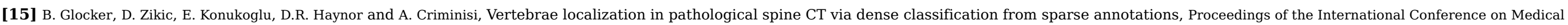
Image Computing and Computer-Assisted Intervention, 2013, Springer, 262-270.

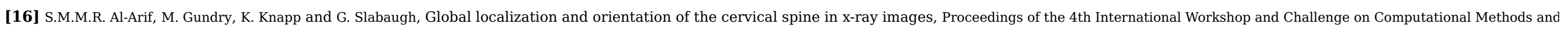
Clinical Applications for Spine Imaging, CSI 2016, held in Conjunction with MICCAI 2016, Athens, Greece, October 17, 2016, 10182, 2017, Springer, 64.Revised Selected Papers

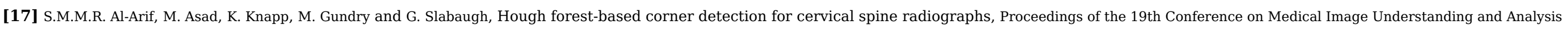
(MIUA), 2015, 183-188.

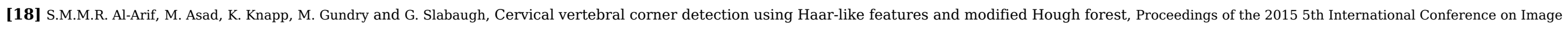
Processing Theory, Tools and Applications (IPTA), 2015, IEEE.

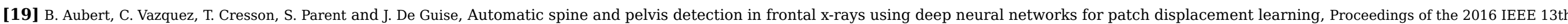
International Symposium on Biomedical Imaging (ISBI), 2016, IEEE, 1426-1429.

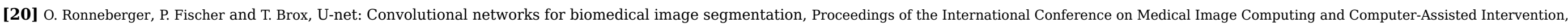
2015, Springer, 234-241.

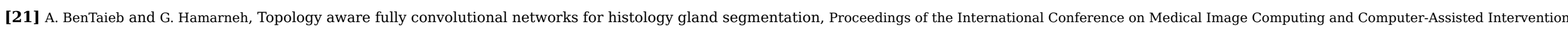
2016, Springer, 460-468.

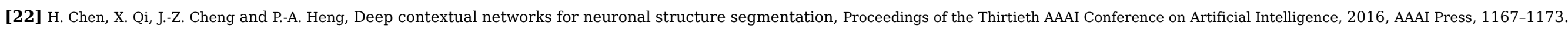

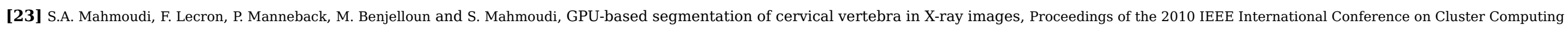
Workshops and Posters (Cluster workshops), 2010, IEEE, 1-8.

[24] S. Ruder, An Overview of Gradient Descent Optimization Algorithms, arXiv preprint arXiv:1609.04747 (2016).

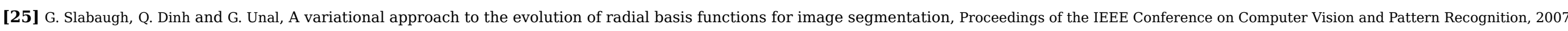
(CVPR'07), 2007, IEEE, 1-8.

[26] H. Noh, S. Hong and B. Han, Learning deconvolution network for semantic segmentation, Proceedings of the IEEE International Conference on Computer Vision, 2015, 1520-1528.

[27] A. Bhattachayya, On a measure of divergence between two statistical population defined by their population distributions, Bull. Calcutta Math. Soc. 35 (99-_109), 1943, 28.

[28] Y. Benjamini and Y. Hochberg, Controlling the false discovery rate: a practical and powerful approach to multiple testing, J. R. stat. Soc. Ser. B (Methodol.) 1995, 289-300.

[29] Convert Region of Interest Polygon to Region Mask, (https://www.mathworks.com/help/images/ref/poly2mask.html\#f6-465457/), Accessed: 2017-09-18.

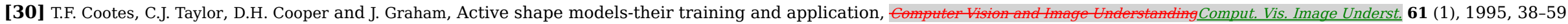

\section{Highlights}

- A deep segmentation network based spine localization algorithm which outperforms the previous state-of-the-art by a large margin.

- A novel spatial probability prediction deep convolutional network which achieves human-level performance in localizing vertebrae centers.

- A novel shape-aware deep segmentation network for vertebrae segmentation. 


\section{Queries and Answers}

Query: Please confirm that givennames and surnames have been identified correctly.

Answer: Yes

Query: Please validate affiliation "b".

Answer: correct

Query: Please validate if corresponding author has been correctly identified.

Answer: Identified correctly.

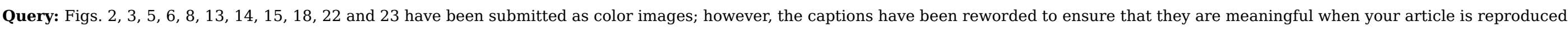
both in color and in black and white. Please check and correct if necessary.

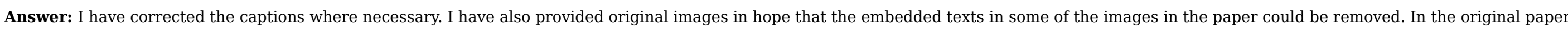

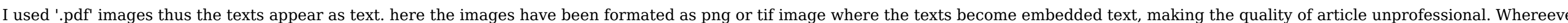
possible, please, update the images with the provided replacement images and add the subfigure indexes appropriately.

Query: Please provide year information in Refs. [1] and [29].

Answer: These are web-links. They do not have a publication year. The date when these web-links have been checked are added in the references as Accessed: xxxx-xx-xx.

Query: Please complete and update Ref. [24].

Answer: This is an unpublished work only available as arXiv preprint. It has already been cited in 151 times.

Query: Please provide volume number in Ref. [28].

Answer: No volume number available 